\title{
Efficient synthesis of 2-alkyl-2-hydroxy-6-X-1-tetralones
}

\author{
Marek Pažický, Vladislav Semak, Boris Gášpár, Adela Bílešová, Marta Sališová,* and \\ Andrej Boháč
}

Comenius University in Bratislava, Faculty of Natural Sciences, Department of Organic

Chemistry, Mlynská dolina CH-2, 84215 Bratislava, Slovakia

E-mail: salisova@,fns.uniba.sk

\section{Dedicated to Professor Arlette Solladié-Cavallo on her $70^{\text {th }}$ anniversary}

\begin{abstract}
Synthesis of 2-alkyl-2-hydroxy-6-X-1-tetralones by oxidation of 2-alkyl-6-X-1-tetralones with oxygen or air is described. The oxidation is performed in phase-transfer conditions (TEBA) with utilization of $\mathrm{P}\left(\mathrm{OCH}_{3}\right)_{3}$. Convenient synthesis of 2-alkyl-6-X-1-tetralones via 2methoxycarbonyl-6-X-1-tetralone is also described.
\end{abstract}

Keywords: 2-Alkyl-6-X-1-tetralone, dimethylcarbonate (DMC), 2-alkyl-2-hydroxy-6-X-1tetralone, phase-transfer conditions, TEBA, $\mathrm{P}\left(\mathrm{OCH}_{3}\right)_{3}$

\section{Introduction}

The possibility to use 2-hydroxy-2-methyl-1-tetralone (5) as chiral auxiliary for the epimerization or deracemization of alpha-amino acids was presented in our previous papers ${ }^{1}$. The synthesis of 2-hydroxy-2-methyl-1-tetralone (5) in racemic ${ }^{2}$ as well as in enantiomerically enriched form $^{3}$ has been already described. Furthermore, the synthesis of the prerequisite 2-methyl-1-tetralone (4) is quite demanding ${ }^{4}$.

The simplest method for the synthesis of 2-methyl-1-tetralone is direct methylation of 1tetralone, under basic conditions. Unfortunately, this synthesis is accompanied by the formation of dimethylated product, which needs to be separated from monomethylated one (with very similar $\mathrm{R}_{\mathrm{f}}$ ) by column chromatography ${ }^{5}$. Another method leading to almost quantitative yield of 2-metyl-1-tetralone (2) is the reaction using bulky and expensive phosphazene base for abstraction of the proton from 1-tetralone ${ }^{2}$. Recently we published another suitable method for preparation of 2 via Friedel-Crafts reaction of benzene with $\alpha$-methyl- $\gamma$-butyrolactone ${ }^{6}$.

In this paper, we describe efficient and economical methods for the synthesis of 2-alkyl-6$\mathrm{X}$-1-tetralones and their oxidation to 2-alkyl-2-hydroxy-6-X-1-tetralones by oxygen or air, under phase-transfer conditions (TEBA) with utilization of $\mathrm{P}\left(\mathrm{OCH}_{3}\right)_{3}$. 


\section{Results and Discussion}

Inspired by the reports of Loewenthal ${ }^{7}$ and Brown et $\mathrm{al}^{8}$ we have carried out the synthesis of 2-alkyl-6-X-1-tetralones via 2-methoxycarbonyl-6-X-1-tetralone (Scheme 1, Table 1).

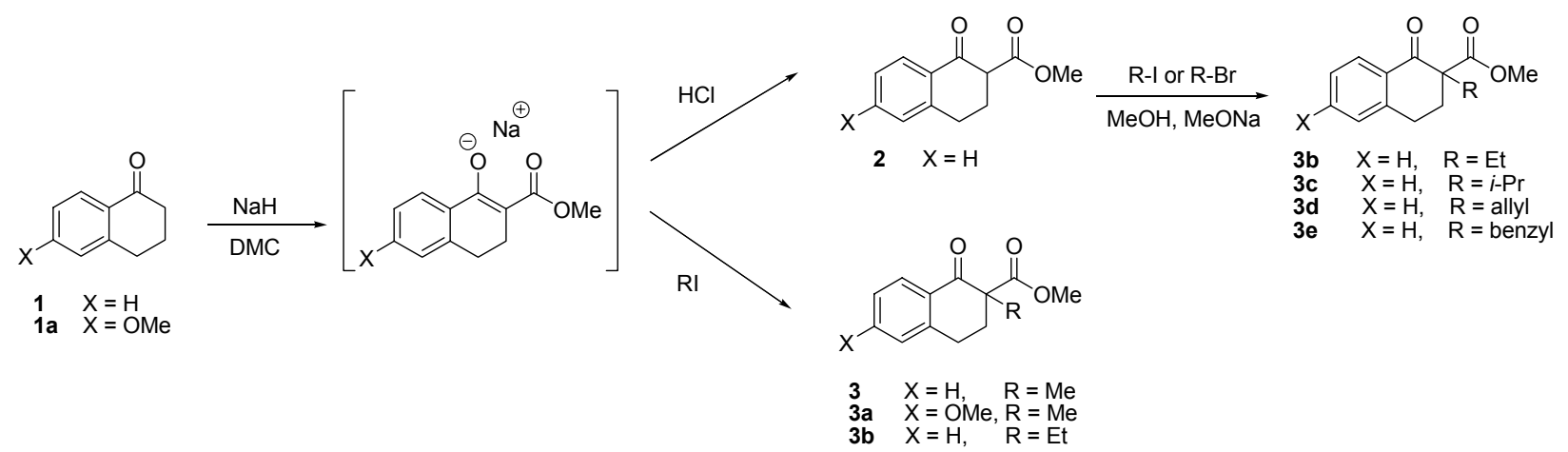

\section{Scheme 1}

Sodium salt of 2-methoxycarbonyl-6-X-1-tetralone (2) was prepared from the corresponding tetralone and DMC under basic conditions. Products (3-3e) obtained after alkylation of 2methoxycarbonyl-6-X-1-tetralones were obtained in varying yields. This diversity was due to the differential reactivity of the alkyl halide used. While methyl and ethyl derivatives had been prepared in almost quantitative yield, alkylation of corresponding ester with more bulky alkyl halides in methanol diminished the yield considerably. Alkylation with allyl bromide gave 52\% yield of compound 3d. While ethylation of $\mathbf{2}$ in methanol gave almost quantitative yield, the best yield of 3c was rather low - 28\%. What more, in these conditions formation of O-alkylated product O-3c was in some cases observed (Figure 1).<smiles>COC(=O)C1=C(OC(C)C)c2ccccc2CC1</smiles>

\section{Figure 1}

Alkylation with benzyl bromide in the same conditions did not give the desired product $3 \mathbf{e}$. Under these conditions, formation of benzylmethylether was observed. Reasonable results were obtained by changing the solvent and reaction conditions. When the benzylation was carried out under phase-transfer conditions (TEBA, toluene), the desired product $\mathbf{3 e}$ was isolated in $77 \%$ yield. Similar procedure was reported by Park et al. ${ }^{9}$ 
Table 1. Preparation of 2-alkyl-2-methoxycarbonyl-6-X-1-tetralones (3-3e)

\begin{tabular}{ccc}
\hline Entry & $\mathrm{R}-\mathrm{X}$ & Yield \\
\hline 1 & $\mathrm{MeI}$ & $\mathbf{3}(98 \%)^{\mathrm{a}}$ \\
2 & $\mathrm{MeI}$ & $\mathbf{3 a}(98 \%)^{\mathrm{a}}$ \\
3 & $\mathrm{EtI}$ & $\mathbf{3 b}(99 \%)^{\mathrm{b}}$ \\
4 & $i-\mathrm{PrBr}$ & $\mathbf{3 c}(28 \%)^{\mathrm{b}}$ \\
5 & $\mathrm{AllylBr}$ & $\mathbf{3 d}(52 \%)^{\mathrm{b}}$ \\
6 & $\mathrm{BnBr}$ & $\mathbf{3 e}(77 \%)^{\mathrm{c}}$ \\
\hline
\end{tabular}

Reagent and conditions: (a) General method I, NaH in DMC; (b) General method II, NaOMe in $\mathrm{MeO}$;. (c) Phase transfer conditions.

The 2-alkyl-1-tetralones (4-4e) were prepared via hydrolysis and decarboxylation of the corresponding esters 3-3e. Under acidic conditions, the corresponding 2-alkylated-1-tetralones were directly prepared. We also found that basic hydrolysis followed by acidification and decarboxylation were in some cases more efficient (see experimental part). Krapcho described the dealkoxycarbonylation of $\beta$-keto esters in wet DMSO, containing sodium chloride ${ }^{10}$.

As the oxidation of racemic 2-methyl-1-tetralone (4) by $m$-CPBA is rather expensive and risky, we decided to examine the oxidation of the corresponding 2-alkyl-1-tetralones $4-4 \mathbf{e}$ with oxygen or air, under phase-transfer conditions (TEBA) using $\mathrm{P}\left(\mathrm{OCH}_{3}\right)_{3}$ inspired by Masui ${ }^{3 \mathrm{c}}$. Various 2-alkyl-2-hydroxy-6-X-1-tetralones (5-5e) are easily accessible following the method reported here (Scheme 2, Table 2). In some cases, the reaction takes a long time.

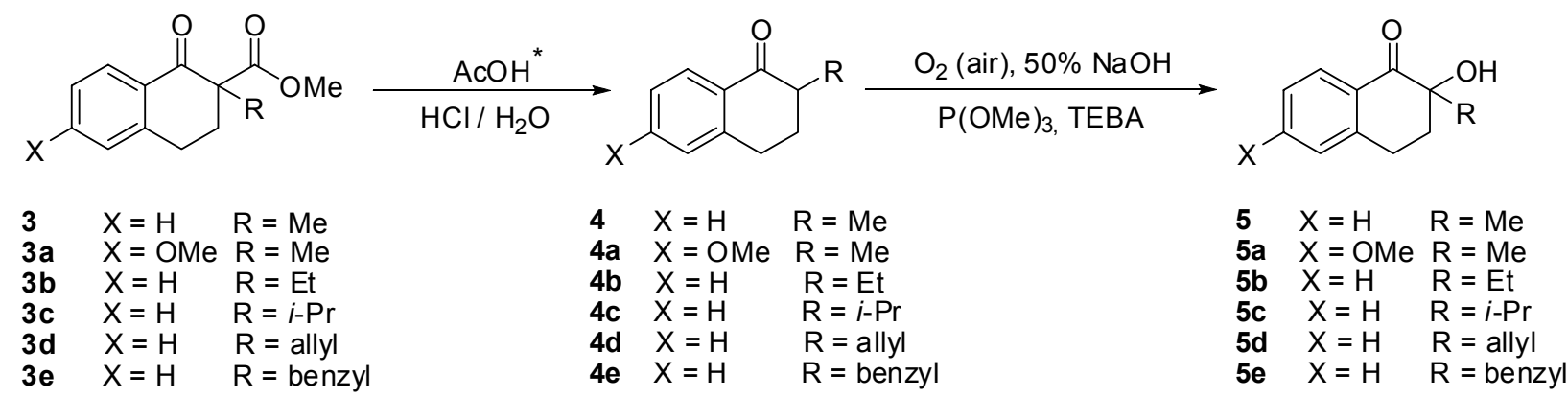

* in some cases basic hydrolysis was more efficient

\section{Scheme 2}

Similar procedures using chiral phase transfer catalysts are also known from literature ${ }^{3}$. Hence, enantioselective oxidation by air to obtain the corresponding hydroxy ketone can be readily carried out by chiral crown ether catalyst as described by Vries et al. ${ }^{11}$ 
Table 2. Preparation of 2-alkyl-2-hydroxy-6-X-1-tetralones

\begin{tabular}{cccc}
\hline Entry & Substrate & Decarboxylation (Yield) & PTC Oxidation (Yield) \\
\hline 1 & $\mathbf{3}$ & $\mathbf{4}(96 \%)^{\mathrm{a}}$ & $\mathbf{5}(95 \%)$ \\
2 & $\mathbf{3 a}$ & $\mathbf{4 a}(98 \%)^{\mathrm{a}}$ & $\mathbf{5 a}(49 \%)$ \\
3 & $\mathbf{3 b}$ & $\mathbf{4 b}(65 \%)^{\mathrm{a}}$ & $\mathbf{5 b}(78 \%)$ \\
4 & $\mathbf{3 c}$ & $\mathbf{4 c}(86 \%)^{\mathrm{a}}$ & $\mathbf{5 c}(50 \%)$ \\
5 & $\mathbf{3 d}$ & $\mathbf{4 d}(87 \%)^{\mathrm{b}}$ & $\mathbf{5 d}(95 \%)$ \\
6 & $\mathbf{3 e}$ & $\mathbf{4 e}(93 \%)^{\mathrm{b}}$ & $\mathbf{5 e}(89 \%)$ \\
\hline
\end{tabular}

Reagent and conditions: (a) acidic hydrolysis and decarboxylation; (b) basic hydrolysis conditions followed by decarboxylation and acidification.

\section{Conclusions}

In conclusion, we developed a valuable methodology for the synthesis of 2-alkyl-1-tetralones and 2-alkyl-2-hydroxy-1-tetralones by alkylation of 2-metoxycarbonyl-1-tetralone, subsequent COOMe removing and mild oxidation under PTC conditions. The synthetic method described here avoids difficulties encountered in direct alkylation of tetralones enolate.

\section{Experimental Section}

General Procedure. ${ }^{1} \mathrm{H}$ and ${ }^{13} \mathrm{C}$ NMR spectra were measured on Varian Gemini (300 MHz and $75 \mathrm{MHz}$ ) apparatus. Chemical shifts are stated in ppm and tetramethysilane (TMS) was used as internal standard. The course of the reactions was monitored by TLC (Merck Silica gel $60 \mathrm{~F}_{254}$ or SILUFOL-UV254); for visualization an UV lamp $254 \mathrm{~nm}$ and iodine vapor or $10 \%$ solution of phosphomolybdic acid in EtOH were used. For column chromatography silica gel Merck 60 (40$63 \mu \mathrm{m})$ was employed. Melting points were determined on a Kofler apparatus and are not corrected. Infrared spectra were measured on an apparatus FT-IR-ATR REACT IR 1000 (ASI Applied Systems) with a diamond probe and MTS detector.

The solvents $\mathrm{CH}_{2} \mathrm{Cl}_{2}, \mathrm{MeCN}$ and $\mathrm{MeOH}$ were dried by distillation from $\mathrm{CaH}_{2}$. Benzene, Hexsol ("C6" fractions $35-100{ }^{\circ} \mathrm{C}$, Microchem) and THF were dried by distillation from sodium. Purification of solvents was performed in accordance with usual methods ${ }^{12} .1$-Tetralone (1) and 6-methoxy-1-tetralone (1a), commercially available (Acros), were distilled before use.

Preparation of Methyl 1,2,3,4-tetrahydro-1-oxonaphthalene-2-carboxylate (2). (2Methoxycarbonyl-1-tetralone). 1-Tetralone (1) $(2.8 \mathrm{~mL}, 20.88 \mathrm{mmol}, 1.0$ equiv.) was added dropwise (during $15 \mathrm{~min}$ ) to a vigorously stirred suspension of $\mathrm{NaH}$ (60\% dispersion in oil, 900 
mg, 22.50 mmol, 1.1 equiv.) in freshly dried and distilled DMC (20 mL, 237 mmol, 11.3 equiv.), under nitrogen atmosphere. The reaction mixture was heated to reflux for $20 \mathrm{~min}$ while a lilacwhite solid was formed and evolvement of hydrogen was observed. After this period the reaction mixture stiffed. [Warning: This experiment has to be set in a flask with higher capacity because of gas evolution; e. g. a $250 \mathrm{~mL}$ round bottomed flask was used in this experiment.] The solid was allowed to cool down to $\mathrm{rt}$, dissolved in hydrochloric acid ( $1 \mathrm{M}, 60 \mathrm{~mL}$, to $\mathrm{pH} \approx 5)$ and extracted with EtOAc (4 x $50 \mathrm{~mL})$. Combined extracts were dried $\left(\mathrm{Na}_{2} \mathrm{SO}_{4}\right)$, filtered and concentrated in vacuo to yield a brown solid (4.5 g). [Ratio of keto : enol tautomers 34 : 66 was determined by ${ }^{1} \mathrm{H}-\mathrm{NMR}$ of crude material.] This crude product was purified by column chromatography (short column of $\mathrm{SiO}_{2}$, Hexsol:EA - 5:1) to yield a white crystalline solid 2 (4.4 g, 97\%) m.p. $73.0-77.4{ }^{\circ} \mathrm{C}$. Brown ${ }^{8}$ refers m.p. $61.5-69.5^{\circ} \mathrm{C}$.

[Note: As it was expected, separation of tautomers was not possible, just enriched fractions were collected for spectral analysis.]

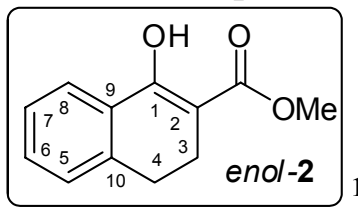

${ }^{1} \mathbf{H}-\mathbf{N M R}\left(300 \mathrm{MHz}, \mathrm{CDCl}_{3}, \delta\right)$ [keto : enol tautomer = $\left.11: 89\right]$ enol form: $12.40(\mathrm{~s}, 1 \mathrm{H}, \mathrm{OH}) ; 7.80\left(\mathrm{dd}, 1 \mathrm{H},{ }^{4} J(8,6)=1.7,{ }^{3} J(8,7)=7.4 \mathrm{~Hz}, \mathrm{H}-(\mathrm{C} 8)\right) ; 7.28-7.33(\mathrm{~m}, 2 \mathrm{H}, \mathrm{H}-$ $(\mathrm{C} 6)+\mathrm{H}-(\mathrm{C} 7)) ; 7.17\left(\mathrm{dd}, 1 \mathrm{H},{ }^{4} J(5,7)=0.8,{ }^{3} J(5,6)=7.3 \mathrm{~Hz}, \mathrm{H}-(\mathrm{C} 5)\right) ; 3.83\left(\mathrm{~s}, 3 \mathrm{H}, \mathrm{CH}_{3} \mathrm{O}-\right)$; $2.82\left(\mathrm{t}, 2 \mathrm{H},{ }^{3} J(3,4)=7.2 \mathrm{~Hz}, 2 \times \mathrm{H}-(\mathrm{C} 4)\right) ; 2.57(\mathrm{t}, 2 \mathrm{H}, J(3,4)=7.2 \mathrm{~Hz}, 2 \times \mathrm{H}-(\mathrm{C} 3))$.

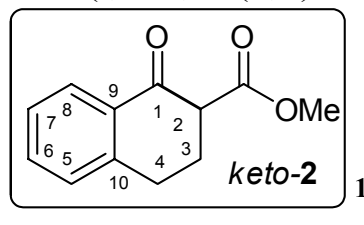

${ }^{1} \mathbf{H}-\mathbf{N M R}\left(300 \mathrm{MHz}, \mathrm{CDCl}_{3}, \delta\right)[$ keto $:$ enol $=35: 65]$ keto form: 8.05 (dd, $\left.1 \mathrm{H},{ }^{4} J(6,8)=1.5,{ }^{3} J(7,8)=7.8 \mathrm{~Hz}, \mathrm{H}-(\mathrm{C} 8)\right) ; 7.50\left(\mathrm{dt}, 1 \mathrm{H},{ }^{4} J(6,8)=1.5,{ }^{3} J=7.5 \mathrm{~Hz}, \mathrm{H}-(\mathrm{C} 6)\right)$; 7.24-7.35 (m, 2H, 2 x H-Ar)); 3.79 (s, 3H, $\left.\mathrm{CH}_{3} \mathrm{O}-\right) ; 3.63$ (dd, $1 \mathrm{H},{ }^{3} J=4.8,{ }^{3} J=10.2 \mathrm{~Hz}, \mathrm{H}-(\mathrm{C} 2)$ ); 2.94- 3.13 (m, 2H, 2 x H-(C4)); 2.45-2.58 (m, 1H, H-(C3)); 2.32-2.41 (m, 1H, H-(C3)). ${ }^{13} \mathrm{C}-$ NMR $\left(75 \mathrm{MHz}, \mathrm{CDCl}_{3}, \delta\right.$ ) [just signals for keto form were detected; probably (when the sample was left in $\mathrm{CDCl}_{3}$ ) equilibrium was markedly shifted to keto form]: $193.10(\mathrm{C}=\mathrm{O}, \mathrm{C} 1), 170.60$ (COO), 143.62 (C10-Ar), 133.89 (C6), 131.67 (C9-Ar), 128.78 (CHAr), 127.74 (CHAr), 126.89 (CHAr), 54.41 (C2), $52.33\left(\mathrm{CH}_{3} \mathrm{O}-\right), 27.59$ (C4), 26.34 (C3). IR: $\left(\mathrm{CHCl}_{3}, \mathrm{~cm}^{-1}\right): 2975$ (m, C$\mathrm{H}_{\text {alif.) }}, 1740$ (s, ester $\mathrm{C}=\mathrm{O}$, ), 1675 (s, $\alpha$-aryl ketone $\left.\mathrm{C}=\mathrm{O}\right), 1455$ (m), 1312 (s), 1215 (s), 1151 (s), 737 (s, C-H arom.).

Infrared, ${ }^{1} \mathrm{H}$ NMR and ${ }^{13} \mathrm{C}$ NMR spectral data are in agreement with those reported previously. ${ }^{8}$

Preparation of Methyl 2-alkyl-1,2,3,4-tetrahydro-1-oxonaphthalene-2-carboxylates (3-3e). (2-Alkyl-2-methoxycarbonyl-1-tetralones)

General procedure I - according to the procedure of Loewenthal ${ }^{7}$. Two-necked flask with magnetic stirring bar, short column and small reflux-distillation head was flamed out under argon 
atmosphere. After cooling $\mathrm{NaH}(95 \%, 2.21 \mathrm{~g}, 87.50 \mathrm{mmol}, 1.17$ equiv.) was added to the anhyd. DMC (210 mL). To the formed suspension 1-tetralone (1) was added (10.2 mL, $74.65 \mathrm{mmol}, 1.0$ equiv.) under argon. The reaction was initiated by addition 10 drops of absolute methanol and by warming to $65-70^{\circ} \mathrm{C}$ (oil bath). When hydrogen evolution had subsided the bath temperature was gradually raised and $\mathrm{MeOH}-\mathrm{DMC}$ azeotrope was very slowly distilled off until the distillation temperature remained above $89^{\circ} \mathrm{C}$ and $50 \mathrm{~mL}$ distillate had been collected. The apparatus was then raised from the oil bath and allowed to cool. To the solidified mixture was added alkyl iodide (1.28 equiv.) and the suspension was heated under reflux with stirring for $3 \mathrm{~h}$ and $80 \mathrm{~mL}$ of the distillate was collected. Reaction mixture was poured on the ice and extracted by $\mathrm{Et}_{2} \mathrm{O}(3 \mathrm{x}$ $150 \mathrm{~mL})$ and by $100 \mathrm{~mL}$ of benzene. Combined organic layers were washed with $2 \mathrm{M} \mathrm{NaOH}(80$ $\mathrm{mL}$ ) and saturated $\mathrm{NaCl}$ solution, dried over $\mathrm{Na}_{2} \mathrm{SO}_{4}$ and concentrated.

General procedure II. To the stirred solution of $\beta$-keto ester 2 (1.0 g, $4.9 \mathrm{mmol}, 1.0$ equiv.) in absolute $\mathrm{MeOH}(20 \mathrm{~mL})$ was added $\mathrm{MeONa}(5.63 \mathrm{mmol}, 1.15$ equiv.) and the reaction mixture was heated to reflux for $30 \mathrm{~min}$. After cooling to $\mathrm{rt}$ alkyl iodide (7.35 mmol, $1.5 \mathrm{equiv}$.) was added and the mixture was refluxed for further $18 \mathrm{~h}$. After cooling to $\mathrm{rt}$ reaction mixture was poured into water and extracted by $\mathrm{CHCl}_{3}(3 \times 15 \mathrm{~mL})$. After common workup desired product was isolated.

General procedure III (inspired by Park et al. $\left.{ }^{\mathbf{9}}\right)$. To a stirred mixture of $\beta$-keto ester $\mathbf{2}$ (1.0 g, $4.90 \mathrm{mmol}, 1.0$ equiv.) in toluene $(50 \mathrm{~mL})$ alkyl halide $(8.36 \mathrm{mmol}, 1.7$ equiv.), $\mathrm{KOH}$ (notch; $1.93 \mathrm{~g}, 34.38 \mathrm{mmol}, 7.0$ equiv.), and TEBA (130 mg, $0.57 \mathrm{mmol}, 0.12$ equiv.) were added at rt. The reaction mixture was vigorously stirred at $\mathrm{rt}$ overnight $(17.5 \mathrm{~h})$. Reaction mixture was diluted with saturated $\mathrm{NH}_{4} \mathrm{Cl}$ solution $(20 \mathrm{~mL})$ and extracted with EtOAc $(3 \times 20 \mathrm{~mL})$. Combined organic layers were dried over $\mathrm{Na}_{2} \mathrm{SO}_{4}$, filtered, concentrated and purified by column chromatography.

Methyl

2-methyl-1,2,3,4-tetrahydro-1-oxonaphthalene-2-carboxylate

(3). (2-Methoxycarbonyl-2-methyl-1-tetralone). Compound $\mathbf{3}$ was prepared according to the "General procedure I" and isolated as colorless oil in $98 \%$ yield.

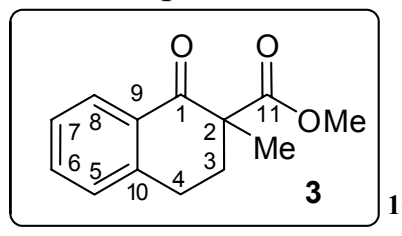

${ }^{1} \mathbf{H}$ NMR $\left(\mathrm{CDCl}_{3}, 300 \mathrm{MHz}, \delta\right): 8.07(\mathrm{dd}, 1 \mathrm{H}, J(7,8)=8.0, J(6,8)=1.5$, $\mathrm{H}-(\mathrm{C} 8)) ; 7.48(\mathrm{ddd}, 1 \mathrm{H}, J(5,6)=8.0, J(6,7)=7.5, J(6,8)=1.5, \mathrm{H}-(\mathrm{C} 6)) ; 7.32(\mathrm{dd}, 1 \mathrm{H}, J(7,8)=$ $8.0, J(6,7)=7.5, \mathrm{H}-(\mathrm{C} 7)) ; 7.22(\mathrm{~d}, 1 \mathrm{H}, J(5,6)=7.5, \mathrm{H}-(\mathrm{C} 5)) ; 3.68$ (s, 3H, MeO-(C11)); 3.05 $\left(\mathrm{ddd}, 1 \mathrm{H},{ }^{2} J=17.5, J(3,4)=9.5, J(3,4)=5.0, \mathrm{H}-(\mathrm{C} 4)\right) ; 2.93\left(\mathrm{ddd}, 1 \mathrm{H},{ }^{2} J=17.5, J(3,4)=5.5\right.$, $J(3,4)=5.0, \mathrm{H}-(\mathrm{C} 4)) ; 2.61\left(\mathrm{ddd}, 1 \mathrm{H},{ }^{2} J=13.5, J(3,4)=9.5, J(3,4)=5.0, \mathrm{H}-(\mathrm{C} 3)\right) ; 2.61(\mathrm{ddd}, 1 \mathrm{H}$, $\left.{ }^{2} J=13.5, J(3,4)=5.5, J(3,4)=5.0, \mathrm{H}-(\mathrm{C} 3)\right) ; 1.51(\mathrm{~s}, 3 \mathrm{H}, \mathrm{Me}-(\mathrm{C} 2)) .{ }^{13} \mathbf{C ~ N M R}\left(\mathrm{CDCl}_{3}, 75 \mathrm{MHz}\right.$, $\delta): 196.26$ (C1), 173.62 (C11), 143.39 (C10), 133.69 (CH), 131.82 (C9), 128.94 (CH), 128.28 
(CH), $127.01(\mathrm{CH}), 54.09$ (MeO-(C11)), 52.69 (C2), 34.17 (C3), 26.23 (C4), 20.96 (Me-(C2)). IR $\left(\mathrm{CHCl}_{3}, \mathrm{~cm}^{-1}\right): 1730(\mathrm{~s}, \mathrm{O}-\mathrm{C}=\mathrm{O}), 1682(\mathrm{~s}, \mathrm{C}=\mathrm{O}), 1600(\mathrm{~m}, \mathrm{C}=\mathrm{C}), 1425(\mathrm{~m}), 1373(\mathrm{w}), 1304$ $(\mathrm{w}), 1212(\mathrm{~s}), 921(\mathrm{~m}), 760$ (s). Elemental analysis $\left(\mathrm{C}_{13} \mathrm{H}_{14} \mathrm{O}_{3}\right)$ : calc. $\mathrm{C} 71.54 \%$; $6.47 \%$; found $\mathrm{C} 71.03 \%$; $\mathrm{H} 6.36 \%$.

Infrared, ${ }^{1} \mathrm{H}$ NMR and ${ }^{13} \mathrm{C}$ NMR spectral data are in agreement with those reported previously. ${ }^{13}$

Methyl 6-methoxy-2-methyl-1,2,3,4-tetrahydro-1-oxonaphthalene-2-carboxylate (3a). (6-Methoxy-2-methoxycarbonyl-2-methyl-1-tetralone). Compound 3a was prepared according to the "General procedure I" and isolated as white crystalline product, m.p. $89-91^{\circ} \mathrm{C}$, in $98 \%$ yield. Mandal ${ }^{14}$ refers m.p. $92{ }^{\circ} \mathrm{C}$.

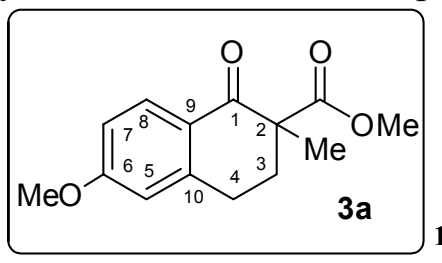

${ }^{1} \mathbf{H}$ NMR $\left(\mathrm{CDCl}_{3}, 300 \mathrm{MHz}, \delta\right): 8.04\left(\mathrm{~d}, 1 \mathrm{H},{ }^{3} \mathrm{~J}=8.7 \mathrm{~Hz}, \mathrm{H}-(\mathrm{C} 8)\right)$; $6.84\left(\mathrm{dd}, 1 \mathrm{H},{ }^{3} \mathrm{~J}=8.8 \mathrm{~Hz},{ }^{4} \mathrm{~J}=2.7 \mathrm{~Hz}, \mathrm{H}-(\mathrm{C} 7)\right) ; 6.67$ (d, 1H, $\left.{ }^{4} \mathrm{~J}=2.4 \mathrm{~Hz}, \mathrm{H}-(\mathrm{C} 5)\right) ; 3.86$ (s, 3H, (C6)-OCH $H_{3}$ ); 3.86 (s, 3H, (C11)-OCH $H_{3}$; 2.95 (ddd, $2 \mathrm{H}, 2 \mathrm{x}^{3} J=4.5 \mathrm{~Hz},{ }^{3} J=9.45 \mathrm{~Hz}, 2 \mathrm{x}^{2} J=$ $\left.17.1 \mathrm{~Hz},{ }^{3} J=5.7 \mathrm{~Hz}, 2 \times \mathrm{H}-(\mathrm{C} 4)\right) ;, 2.59\left(\mathrm{ddd}, 1 \mathrm{H},{ }^{3} J=5.7 \mathrm{~Hz},{ }^{3} J=4.5 \mathrm{~Hz},{ }^{2} J=13.5 \mathrm{~Hz}, \mathrm{H}-\right.$ (C3)); 2.03 (ddd, $1 \mathrm{H},{ }^{3} J=4.8 \mathrm{~Hz},{ }^{3} J=9.45 \mathrm{~Hz},{ }^{2} J=13.7 \mathrm{~Hz}, \mathrm{H}-(\mathrm{C} 3)$ ); 1.50 (s, 3H, $-\mathrm{CH}_{3}$ ).

${ }^{1} \mathrm{H}$ NMR spectral data are in agreement with those reported previously. ${ }^{14}$

Methyl 2-ethyl-1,2,3,4-tetrahydro-1-oxonaphthalene-2-carboxylate (3b). (2-Ethyl-2methoxycarbonyl-1-tetralone). By the reaction conditions described in "General procedure I" only $25 \%$ yield of compound $\mathbf{3 b}$ was determined in the reaction mixture according to the ${ }^{1} \mathrm{H}$ NMR. Reaction mixture contained also $\mathbf{3 b}$ and starting ester $\mathbf{2}$ in both - keto-enol forms. Separation of this mixture by chromatography was not effective. Therefore all fractions were collected, solvent evaporated and the crude mixture was used for further ethylation according to the „General procedure II“".

Reaction mixture was dissolved in anhydrous $\mathrm{MeOH}$ and excess of in situ prepared MeONa was added. This mixture was heated to relux for $30 \mathrm{~min}$, then cooled to $\mathrm{rt}$ and excess of ethyl iodide was added. The reaction mixture was monitored by TLC and quenched after $20 \mathrm{~h}$ when starting material was not present any more. After cooling to $\mathrm{rt}$ the reaction mixture was poured into water and extracted with $\mathrm{Et}_{2} \mathrm{O}$. Combined organic layers were dried $\left(\mathrm{Na}_{2} \mathrm{SO}_{4}\right)$, filtered and concentrated. By this way almost quantitative yield of the desired product $\mathbf{3} \mathbf{b}$ was isolated as reddish-brown oil.

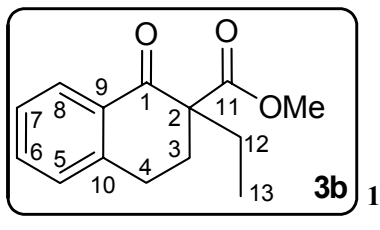

${ }^{1} \mathbf{H}$ NMR $\left(\mathrm{CDCl}_{3}, 300 \mathrm{MHz}, \delta\right): 8.05(\mathrm{dd}, 1 \mathrm{H}, J(7,8)=7.5, J(6,8)=1.5, \mathrm{H}-$ $(\mathrm{C} 8)) ; 7.47(\mathrm{ddd}, 1 \mathrm{H}, J(5,6)=7.5, J(6,7)=7.5, J(6,8)=1.5, \mathrm{H}-(\mathrm{C} 6)) ; 7.31(\mathrm{ddd}, 1 \mathrm{H}, J(6,7)=7.5$, 
$J(7,8)=7.5, J(5,7)=1.0, \mathrm{H}-(\mathrm{C} 7)) ; 7.22(\mathrm{dd}, 1 \mathrm{H}, J(5,6)=7.5, J(5,7)=1.0, \mathrm{H}-(\mathrm{C} 5)) ; 3.68(\mathrm{~s}, 3 \mathrm{H}$, MeO-(C11)); $3.08\left(\mathrm{ddd}, 1 \mathrm{H},{ }^{2} J=17.5, J(3,4)=10.0, J(3,4)=5.0, \mathrm{H}-(\mathrm{C} 4)\right) ; 2.92\left(\mathrm{ddd}, 1 \mathrm{H},{ }^{2} J=\right.$ $17.5, J(3,4)=5.5, J(3,4)=5.0, \mathrm{H}-(\mathrm{C} 4)) ; 2.56\left(\mathrm{ddd}, 1 \mathrm{H},{ }^{2} J=14.0, J(3,4)=5.5, J(3,4)=5.0, \mathrm{H}-\right.$ $(\mathrm{C} 3)) ; 2.14\left(\mathrm{ddd}, 1 \mathrm{H},{ }^{2} J=14.0, J(3,4)=10.0, J(3,4)=5.0, \mathrm{H}-(\mathrm{C} 3)\right) ; 2.07\left(\mathrm{dq}, 1 \mathrm{H},{ }^{2} J=15.0\right.$, $J(12,13)=7.5, \mathrm{H}-(\mathrm{C} 12)) ; 1.95\left(\mathrm{dq}, 1 \mathrm{H},{ }^{2} J=15.0, J(12,13)=7.5, \mathrm{H}-(\mathrm{C} 12)\right) ; 0.98(\mathrm{t}, 3 \mathrm{H}, J(12,13)$ $=7.5, \mathrm{H}-(\mathrm{C} 13)) .{ }^{13} \mathbf{C}$ NMR $\left(\mathrm{CDCl}_{3}, 75 \mathrm{MHz}, \delta\right): 195.74(\mathrm{C} 1), 172.61(\mathrm{C} 11), 143.35(\mathrm{C} 10)$, $133.62(\mathrm{CH}), 132.21(\mathrm{C} 9), 128.92(\mathrm{CH}), 128.26(\mathrm{CH}), 126.93(\mathrm{CH}), 58.06(\mathrm{C} 2), 52.55(\mathrm{MeO}-$ (C11)), 30.17 (C3), $27.25\left(\mathrm{CH}_{2}\right), 26.09\left(\mathrm{CH}_{2}\right), 9.40(\mathrm{C} 13)$. IR $\left(\mathrm{CHCl}_{3}, \mathrm{~cm}^{-1}\right): 1720(\mathrm{~s}, \mathrm{O}-\mathrm{C}=\mathrm{O})$, $1674(\mathrm{~s}, \mathrm{C}=\mathrm{O}), 1593(\mathrm{~m}, \mathrm{C}=\mathrm{C}), 1435$ (m), $1280(\mathrm{w}), 1280(\mathrm{w}), 1210(\mathrm{~s}), 893$ (w), 766 (s), 689 (w). Elemental analysis $\left(\mathrm{C}_{14} \mathrm{H}_{16} \mathrm{O}_{3}\right)$ : calc. C $72.39 \%$; H $6.94 \%$; found $\mathrm{C} 72.51 \%$; $6.89 \%$.

Methyl 2-isopropyl-1,2,3,4-tetrahydro-1-oxonaphthalene-2-carboxylate (3c). (2-Isopropyl2-methoxycarbonyl-1-tetralone). Reaction was carried out according to the „General procedure II“. After common work-up the crude product (yellow oil) contained only $17 \%$ of the desired product 3c (according to the ${ }^{1} \mathrm{H}$ NMR). What more, also $16 \%$ of $\mathrm{O}$-alkylated product $\mathbf{O}-3 \mathbf{c}$ was present in the crud product and it was impossible to separate it from the product 3c.

Note 1. When the amount of $\mathrm{MeONa}$ increased to 1.5 equiv. and reaction time was prolonged to $40 \mathrm{~h}, \mathrm{O}$-alkylated product $\mathbf{O}-3 \mathbf{c}$ was not present in reaction mixture. In these conditions desired product 3c was isolated in $28 \%$ yield as yellowish oil.

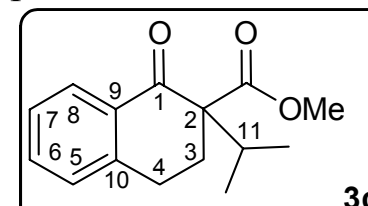

3c ${ }^{1} \mathbf{H}$ NMR $\left(300 \mathrm{MHz}, \mathrm{CDCl}_{3}, \delta\right): 8.05\left(\mathrm{dd}, 1 \mathrm{H},{ }^{3} J=7.5 \mathrm{~Hz},{ }^{4} J=1.5 \mathrm{~Hz}\right.$, $\mathrm{H}-(\mathrm{C} 8)) ; 7.45$ (dt, $\left.1 \mathrm{H},{ }^{3} J=7.5 \mathrm{~Hz},{ }^{4} J=1.5 \mathrm{~Hz}, \mathrm{H}-(\mathrm{C} 6)\right) ; 7.29$ (t, $\left.1 \mathrm{H},{ }^{3} J=7.5 \mathrm{~Hz}, \mathrm{H}-(\mathrm{C} 7)\right) ; 7.20$ $\left(\mathrm{d}, 1 \mathrm{H},{ }^{3} \mathrm{~J}=7.5 \mathrm{~Hz}, \mathrm{H}-(\mathrm{C} 5)\right) ; 3.64\left(\mathrm{~s}, 3 \mathrm{H}, \mathrm{O}-\mathrm{CH}_{3}\right) ; 3.21\left(\mathrm{ddd}, 1 \mathrm{H},{ }^{2} \mathrm{~J}=15.3 \mathrm{~Hz},{ }^{3} \mathrm{~J}=12 \mathrm{~Hz},{ }^{3} J=\right.$ $4.5 \mathrm{~Hz}, \mathrm{H}-(\mathrm{C} 4)) ; 2.89$ (ddd, $1 \mathrm{H},{ }^{2} J=17.1 \mathrm{~Hz},{ }^{3} J=4.5 \mathrm{~Hz},{ }^{3} J=3 \mathrm{~Hz}, \mathrm{H}-(\mathrm{C} 4)$ ); 2.71 (sept., $1 \mathrm{H},{ }^{3} J$ $=6.6 \mathrm{~Hz}, \mathrm{H}-(\mathrm{C} 11)) ; 2.47\left(\mathrm{ddd}, 1 \mathrm{H},{ }^{2} J=15.3 \mathrm{~Hz},{ }^{3} J=5.1 \mathrm{~Hz},{ }^{3} J=3 \mathrm{~Hz}, \mathrm{H}-(\mathrm{C} 3)\right) ; 2.12$ (ddd, $1 \mathrm{H}$, $\left.{ }^{2} J=17.1 \mathrm{~Hz},{ }^{3} J=12 \mathrm{~Hz},{ }^{3} J=5.1 \mathrm{~Hz}, \mathrm{H}-(\mathrm{C} 3)\right) ; 0.99$ (d, 3H, $\left.{ }^{3} J=6.6 \mathrm{~Hz}, \mathrm{CH}_{3}-(i-P r)\right) ; 0.96$ (d, $\left.3 \mathrm{H},{ }^{3} \mathrm{~J}=6.6 \mathrm{~Hz}, \mathrm{CH}_{3}-(\mathrm{i}-\mathrm{Pr})\right) .{ }^{13} \mathrm{C}$ NMR $\left(300 \mathrm{MHz}, \mathrm{CDCl}_{3}, \delta\right): 194.86(\mathrm{C}=\mathrm{O}), 171.57(\mathrm{O}=\mathrm{C}-\mathrm{O})$, 143.81 (C9), 143.81 (C9), $133.58\left(\mathrm{CH}_{\mathrm{Ar}}\right), 132.84(\mathrm{C} 10), 128.90\left(\mathrm{CH}_{\mathrm{Ar}}\right), 128.26\left(\mathrm{CH}_{\mathrm{Ar}}\right), 126.78$ $\left(\mathrm{CH}_{\mathrm{Ar}}\right), 61.19(\mathrm{C} 2), 52.47\left(\mathrm{O}-\mathrm{CH}_{3}\right), 32.70(\mathrm{C} 11), 26.57\left(\mathrm{CH}_{2}\right), 26.26\left(\mathrm{CH}_{2}\right), 18.78\left(\mathrm{CH}_{3}-(i-P r)\right)$, $17.77\left(\mathrm{CH}_{3}-(\mathrm{i}-\mathrm{Pr})\right)$. IR $\left(\mathrm{CHCl}_{3}, \mathrm{~cm}^{-1}\right): 2950\left(\mathrm{~s}, \mathrm{C}_{\mathrm{Ar}}-\mathrm{H}\right), 1740(\mathrm{~m}, \mathrm{O}-\mathrm{C}=\mathrm{O}), 1680(\mathrm{~m}, \mathrm{C}=\mathrm{O}), 1580$ $(\mathrm{w}, \mathrm{C}=\mathrm{C}), 1475(\mathrm{~s}), 1370(\mathrm{~m}), 1275(\mathrm{w}), 1215(\mathrm{~s}, \mathrm{C}-\mathrm{O}), 1135(\mathrm{~m}), 755(\mathrm{~s})$. Elemental analysis $\left(\mathrm{C}_{15} \mathrm{H}_{18} \mathrm{O}_{3}\right)$ : calc. C 73.15\%; $\mathrm{H} 7.37 \%$; found $\mathrm{C} 73.42 \%$; $\mathrm{H} .54 \%$. 


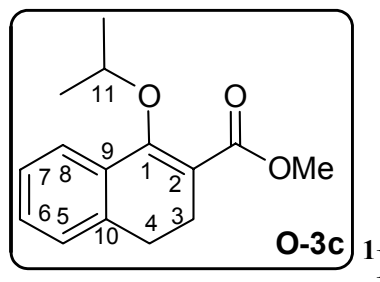

${ }^{1} \mathbf{H}$ NMR spectrum of $\mathbf{O - 3 c}$ was described from the mixed sample containing $70 \%$ of $\mathbf{3 c}$ and $30 \% \mathbf{O - 3 c}$.

${ }^{1} \mathrm{H}$ NMR (300 MHz, $\left.\mathrm{CDCl}_{3}, \delta\right)$ : 8.02-8.05 (m, 1H, H-(C8)); 7.54-7.57 (m, 1H, $\mathrm{H}_{\mathrm{Ar}}$ ); 7.16-7.32 $\left(\mathrm{m}, 2 \mathrm{H}, 2 \mathrm{xH}_{\mathrm{Ar}}\right) ; 4.33$ (sept., $1 \mathrm{H},{ }^{3} J=6 \mathrm{~Hz}, \mathrm{H}-(\mathrm{C} 11)$ ); 3.81 (s, 3H, O-CH $)_{3} ; 2.60-2.78$ (m, 4H, $\left.2 \mathrm{xCH}_{2}\right), 1.31\left(\mathrm{~d}, 6 \mathrm{H},{ }^{3} \mathrm{~J}=6 \mathrm{~Hz}, 2 \mathrm{xCH}-(i-P r)\right)$.

Methyl 2-allyl-1,2,3,4-tetrahydro-1-oxonaphthalene-2-carboxylate (3d). (2-Allyl-2methoxycarbonyl-1-tetralone). The reaction was carried out according to the "General procedure II". After addition of allyl bromide the reaction mixture was heated to reflux for $20 \mathrm{~h}$. After common work-up and chromatography $\left(\mathrm{SiO}_{2}, \mathrm{Hexol}: \mathrm{Et}_{2} \mathrm{O}-4: 1\right) 52 \%$ of desired product 3d was isolated as colorless oil.

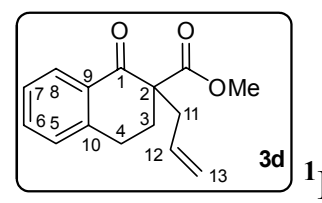

${ }^{1} \mathbf{H}$ NMR $\left(300 \mathrm{MHz}, \mathrm{CDCl}_{3}, \delta\right): 8.05\left(\mathrm{dd}, 1 \mathrm{H},{ }^{3} J=7.8 \mathrm{~Hz},{ }^{4} J=1.2 \mathrm{~Hz}, \mathrm{H}-\right.$ (C8)); 7.47 (dt, $\left.1 \mathrm{H},{ }^{3} J=7.5 \mathrm{~Hz},{ }^{4} J=1.2 \mathrm{~Hz}, \mathrm{H}-(\mathrm{C} 6)\right) ; 7.31\left(\mathrm{t}, 1 \mathrm{H},{ }^{3} J=7.8 \mathrm{~Hz}, \mathrm{H}-(\mathrm{C} 7)\right) ; 7.22$ (d, $\left.1 \mathrm{H},{ }^{3} J=7.5 \mathrm{~Hz}, \mathrm{H}-(\mathrm{C} 5)\right) ; 5.82\left(\mathrm{ddt}, 1 \mathrm{H},{ }^{3} \mathrm{~J}=17.4 \mathrm{~Hz},{ }^{3} \mathrm{~J}=10.2 \mathrm{~Hz},{ }^{3} \mathrm{~J}=7.2 \mathrm{~Hz}, \mathrm{H}-(\mathrm{C} 12)\right)$; 5.09$5.18(\mathrm{~m}, 2 \mathrm{H}, 2 \mathrm{xH}-(\mathrm{C} 13)) ; 3.67\left(\mathrm{~s}, 3 \mathrm{H}, \mathrm{OCH}_{3}\right) ; 3.07$ (ddd, $1 \mathrm{H},{ }^{2} \mathrm{~J}=17.4 \mathrm{~Hz},{ }^{3} \mathrm{~J}=10.2 \mathrm{~Hz},{ }^{3} \mathrm{~J}=4.8$ $\mathrm{Hz}, \mathrm{H}-(\mathrm{C} 4)$ ); 2.92 (ddd, $\left.1 \mathrm{H},{ }^{2} J=17.4 \mathrm{~Hz},{ }^{3} J=5.1 \mathrm{~Hz},{ }^{3} J=5.1 \mathrm{~Hz}, \mathrm{H}-(\mathrm{C} 4)\right) ; 2.75$ (dd, $1 \mathrm{H},{ }^{2} J=$ $\left.13.8 \mathrm{~Hz},{ }^{3} J=7.2 \mathrm{~Hz}, \mathrm{H}-(\mathrm{C} 11)\right) ; 2.68\left(\mathrm{dd}, 1 \mathrm{H},{ }^{2} J=13.8 \mathrm{~Hz},{ }^{3} J=7.2 \mathrm{~Hz}, \mathrm{H}-(\mathrm{C} 11)\right) ; 2.53$ (ddd, $\left.1 \mathrm{H},{ }^{2} J=13.8 \mathrm{~Hz},{ }^{3} J=5.1 \mathrm{~Hz},{ }^{3} J=5.1 \mathrm{~Hz}, \mathrm{H}-(\mathrm{C} 3)\right) ; 2.14\left(\mathrm{ddd}, 1 \mathrm{H},{ }^{2} J=15.3 \mathrm{~Hz},{ }^{3} J=10.2 \mathrm{~Hz},{ }^{3} J\right.$ $=5.1 \mathrm{~Hz}, \mathrm{H}-(\mathrm{C} 3)) .{ }^{13} \mathrm{C}$ NMR $\left(300 \mathrm{MHz}, \mathrm{CDCl}_{3}, \delta\right): 195.14(\mathrm{C}=\mathrm{O}), 172,20(\mathrm{O}-\mathrm{C}=\mathrm{O}), 143.45(\mathrm{C})$, $133.75\left(\mathrm{CH}_{\mathrm{Ar}}\right), 133.56\left(\mathrm{CH}_{\mathrm{Ar}}\right), 132.10(\mathrm{C}), 128.96\left(\mathrm{CH}_{\mathrm{Ar}}\right), 128.30\left(\mathrm{CH}_{\mathrm{Ar}}\right), 126.97(\mathrm{C} 12), 119.13$ $(\mathrm{C} 13), 57.64(\mathrm{C} 2), 52.66\left(\mathrm{OCH}_{3}\right), 68.92\left(\mathrm{CH}_{2}\right), 30.73\left(\mathrm{CH}_{2}\right), 26.04\left(\mathrm{CH}_{2}\right)$. Elemental analysis $\left(\mathrm{C}_{15} \mathrm{H}_{16} \mathrm{O}_{3}\right)$ : calc. C 73.75\%; $\mathrm{H} 6.60 \%$; found $\mathrm{C} 72.33 \%$; $\mathrm{H} 6.63 \%$.

${ }^{1} \mathrm{H}$ NMR and ${ }^{13} \mathrm{C}$ NMR spectral data are in agreement with those reported previously. ${ }^{9}$

Methyl 2-benzyl-1,2,3,4-tetrahydro-1-oxonaphthalene-2-carboxylate (3e). (2-Benzyl-2methoxycarbonyl-1-tetralone). Product 3e was prepared according to the „General procedure III“. After common work-up and purification by column chromatography $\left(\mathrm{SiO}_{2}, \mathrm{Hexsol}_{\mathrm{Et}} \mathrm{O}-\right.$ 5:1) $\alpha$-benzylated $\beta$-keto ester 3e was isolated as a white solid, m.p. $95.3-96.6{ }^{\circ} \mathrm{C}$ in $77 \%$ yield.

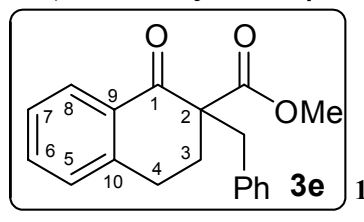

${ }^{1} \mathbf{H}-\mathbf{N M R}\left(300 \mathrm{MHz}, \mathrm{CDCl}_{3}, \delta\right): 8.08\left(\mathrm{dd}, 1 \mathrm{H},{ }^{4} J(8,6)=1.2,{ }^{3} J(8,7)=7.9\right.$ $\mathrm{Hz}, \mathrm{H}-(\mathrm{C} 8)) ; 7.45\left(\mathrm{ddd}, 1 \mathrm{H},{ }^{4} J(6,8)=1.2,{ }^{3} J(6,5)={ }^{3} J(6,7)=7.5 \mathrm{~Hz}, \mathrm{H}-(\mathrm{C} 6)\right) ; 7.15$ (m, 7H, H- 
$(\mathrm{C} 5)+\mathrm{H}-(\mathrm{C} 7)+5 \mathrm{x} \mathrm{H}-(\mathrm{Ph})) ; 3.66\left(\mathrm{~s}, 3 \mathrm{H}, \mathrm{CH}_{3} \mathrm{O}-\right) ; 3.45\left(\mathrm{~d}, 1 \mathrm{H},{ }^{2} \mathrm{~J}=13.6 \mathrm{~Hz},-\mathrm{CH}_{2}-\mathrm{Ph}\right) ; 3.32(\mathrm{~d}$, $\left.1 \mathrm{H},{ }^{2} J=13.6 \mathrm{~Hz},-\mathrm{CH}_{2} \mathrm{-Ph}\right) ; 3.08\left(\mathrm{ddd}, 1 \mathrm{H},{ }^{3} J(3,4)=4.7,{ }^{3} J(3,4)=11.7,{ }^{2} J=16.7 \mathrm{~Hz}, \mathrm{H}-(\mathrm{C} 3)\right)$; $2.85\left(\mathrm{ddd}, 1 \mathrm{H},{ }^{3} J(3,4)=4.3,{ }^{3} J(3,4)=4.7,{ }^{2} J=16.7 \mathrm{~Hz}, \mathrm{H}-(\mathrm{C} 3)\right) ; 2.49\left(\mathrm{ddd}, 1 \mathrm{H},{ }^{3} J(4,3)=4.3\right.$, $\left.{ }^{3} J(4,3)=4.7,{ }^{2} J=13.8 \mathrm{~Hz}, \mathrm{H}-(\mathrm{C} 4)\right) ; 1.99\left(\mathrm{ddd}, 1 \mathrm{H},{ }^{3} J(4,3)=4.7,{ }^{3} J(4,3)=11.7,{ }^{2} J=13.8 \mathrm{~Hz}, \mathrm{H}-\right.$ (C4)). ${ }^{13}$ C-NMR (75 MHz, $\left.\mathrm{CDCl}_{3}, \delta\right): 194.44$ (C1, C=O), 171.83 (COO), 143.25 (C10-Ar), 136.43 (C9-Ar), 133.51 (CHAr), 132.11 (C-Ar, -Ph), 130.68 (2 x CHAr), 128.71 (CHAr), 128.19 (CHAr), 128.12 (2 x CHAr), 126.76 (CHAr), 126.69 (CHAr), 58.72 (C2), 52.56 ( $\left.\mathrm{CH}_{3} \mathrm{O}-\right), 40.14$ (-CH $2-\mathrm{Ph}), 30.39$ (C3), 26.05 (C4). IR $\left(\mathrm{CH}_{2} \mathrm{Cl}_{2}, \mathrm{~cm}^{-1}\right)$ : 2925 (m, C-H $\left.\mathrm{H}_{\text {alif. }}\right), 1746$ (s, ester C=O, ), 1675 (s, $\alpha$-aryl ketone C=O), 1455 (m), 1215 (s), 1151 (s), 737 (s, C-H arom.), 706 (s, C-H $\mathrm{H}_{\text {arom.). }}$. ${ }^{1} \mathrm{H}$ NMR and ${ }^{13} \mathrm{C}$ NMR spectral data are in agreement with those reported previously. ${ }^{9}$

Preparation of 2-alkyl-3,4-dihydronaphthalen-1(2H)-ones (4-4e). (2-alkyl-1-tetralones) General procedure I - acid hydrolysis and decarboxylation. Corresponding 2-alkyl $\beta$-keto ester (3-3e) (73.04 mmol, 1.0 equiv.) was dissolved in the solution containing: glacial acetic acid (127 mL, $2132 \mathrm{mmol}, 28.8$ equiv.), $\mathrm{HCl} 37$ \% (32 mL, $288 \mathrm{mmol}, 3.9$ equiv.) and distilled water $(25 \mathrm{~mL})$. Reaction mixture was heated into reflux and was monitored by TLC. After $4 \mathrm{~h}$ starting material was not present in the reaction mixture. After cooling to the rt reaction mixture was poured into $200 \mathrm{~mL}$ of distilled water and $100 \mathrm{~mL}$ of $\mathrm{CH}_{2} \mathrm{Cl}_{2}$ was added. Neutralization of the solution has been done by slow addition of $96 \mathrm{~g} \mathrm{Na}_{2} \mathrm{CO}_{3}$ (906 mmol, 12.24 equiv.). Water layer was extracted by $\mathrm{CH}_{2} \mathrm{Cl}_{2}(4 \times 100 \mathrm{~mL})$. Combined organic layers were dried over $\mathrm{Na}_{2} \mathrm{SO}_{4}$, filtered and concentrated.

General procedure II - basic hydrolysis and decarboxylation. To the stirred solution of 2alkylated $\beta$-keto ester (3-3e) (2.07 mmol, 1.0 equiv.) in $\mathrm{MeOH} \mathrm{(3} \mathrm{mL)} \mathrm{KOH} \mathrm{(1.16} \mathrm{g,} 20.7 \mathrm{mmol}$, 10 equiv. $)$ in water $(2.3 \mathrm{~mL})$ was added and the reaction mixture was heated to refluxed for 75 min. After cooling to $\mathrm{rt}$ reaction mixture was neutralized by slow addition of $10 \% \mathrm{HCl}$ and extracted by $\mathrm{CH}_{2} \mathrm{Cl}_{2}$ ( $\left.3 \times 20 \mathrm{~mL}\right)$. Combined organic layers were washed with saturated solution of $\mathrm{NaCl}(20 \mathrm{~mL})$, dried over $\mathrm{Na}_{2} \mathrm{SO}_{4}$, filtered and concentrated. Crude product was purified by column chromatography $\left(\mathrm{SiO}_{2}, \mathrm{Hexsol}: \mathrm{Et}_{2} \mathrm{O}-4: 1\right)$.

2-Methyl-3,4-dihydro-2H-naphthalen-1-one (4). (2-Methyl-1-tetralone). Reaction was carried out according to the „General procedure I“. After common work-up crude reaction mixture was distilled on Büchi apparatus $\left(80-130{ }^{\circ} \mathrm{C} / \sim 27 \mathrm{~Pa}\right)$. By this way $11.29 \mathrm{~g}$ (96\%) of colorless oil was isolated.

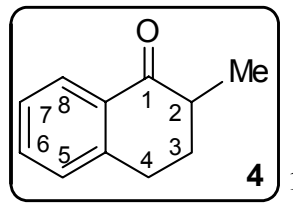

${ }^{1} \mathbf{H}$ NMR $\left(\mathrm{CDCl}_{3}, 300 \mathrm{MHz}, \delta\right): 8.05(1 \mathrm{H}, \mathrm{dd}, J(7,8)=8.0, J(6,8)=1.0, \mathrm{H}-$ $\mathrm{C}(8)) ; 7.47(1 \mathrm{H}, \mathrm{ddd}, J(5,6)=7.5, J(6,7)=7.5, J(6,8)=1.0, \mathrm{H}-\mathrm{C}(6)) ; 7.31(1 \mathrm{H}, \mathrm{dd}, J(7,8)=8.0$, $J(6,7)=7.5, \mathrm{H}-\mathrm{C}(7)) ; 7.25(1 \mathrm{H}, \mathrm{d}, J(5,6)=7.5, \mathrm{H}-\mathrm{C}(5)) ; 3.12-2.91(2 \mathrm{H}, \mathrm{m}$, with measurable 
$J\left(3^{\mathrm{Me}-\mathrm{C}(2)}, 4^{\mathrm{Me}-\mathrm{C}(2)}\right)=11.0, J\left(3^{\mathrm{H}-\mathrm{C}(2)}, 4^{\mathrm{H}-\mathrm{C}(2)}\right)=9.0, J\left(3^{\mathrm{Me}-\mathrm{C}(2)}, 4^{\mathrm{H}-\mathrm{C}(2)}\right)=5.0, J\left(3^{\mathrm{H}-\mathrm{C}(2)}, 4^{\mathrm{Me}-\mathrm{C}(2)}\right) \sim 0.0$, $2 \mathrm{x} \mathrm{H}-\mathrm{C}(4)) ; 2.61\left(1 \mathrm{H}, \mathrm{dqd}, J\left(2,3^{\mathrm{Me}-\mathrm{C}(2)}\right)=12.0, J(\mathrm{Me}, 2)=7.0, J\left(2,3^{\mathrm{H}-\mathrm{C}(2)}\right)=4.5, \mathrm{H}-\mathrm{C}(2)\right) ; 2.21$ $\left(1 \mathrm{H}, \mathrm{ddd},{ }^{2} J=13.0, J\left(3^{\mathrm{H}-\mathrm{C}(2)}, 4^{\mathrm{H}-\mathrm{C}(2)}\right)=9.0, J\left(2,3^{\mathrm{H}-\mathrm{C}(2)}\right)=4.5, J\left(3^{\mathrm{H}-\mathrm{C}(2)}, 4^{\mathrm{Me}-\mathrm{C}(2)}\right) \sim 0.0, \mathrm{H}^{\mathrm{H}-\mathrm{C}(2)}-\right.$ $\mathrm{C}(3)) ; 1.90\left(1 \mathrm{H}, \mathrm{dddd},{ }^{2} J=13.0, J\left(2,3^{\mathrm{Me}-\mathrm{C}(2)}\right)=12.0, J\left(3^{\mathrm{Me}-\mathrm{C}(2)}, 4^{\mathrm{Me}-\mathrm{C}(2)}\right)=11.0, J\left(3^{\mathrm{Me}-\mathrm{C}(2)}, 4^{\mathrm{H}-\mathrm{C}(2)}\right)\right.$ $\left.=5.0, \mathrm{H}^{\mathrm{Me}-\mathrm{C}(2)}-\mathrm{C}(3)\right) ; 1.28(1 \mathrm{H}, \mathrm{d}, J(\mathrm{Me}, 2)=7.0, \mathrm{Me}-\mathrm{C}(2))$.

${ }^{1} \mathrm{H}$ NMR spectral data are in agreement with those reported previously. ${ }^{2}$

6-Methoxy-2-methyl-3,4-dihydronaphthalen-1(2H)-one (4a). (6-Methoxy-2-methyl-1tetralone). Reaction was carried out according to the „General procedure I“. After common work-up the desired product $\mathbf{4 a}$ was isolated as yellow oil in $98 \%$ yield.

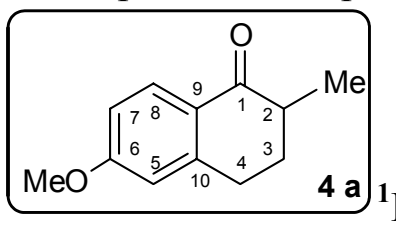

${ }^{1} \mathbf{H}$ NMR $\left(\mathrm{CDCl}_{3}, 300 \mathrm{MHz}, \delta\right) 8.01\left(\mathrm{~d}, 1 \mathrm{H},{ }^{3} J=8.7 \mathrm{~Hz}, \mathrm{H}-(\mathrm{C} 8)\right) ; 6.81$ (dd, $\left.1 \mathrm{H},{ }^{4} J=2.7 \mathrm{~Hz},{ }^{3} J=8.7, \mathrm{H}-(\mathrm{C} 7)\right) ; 6.68\left(\mathrm{~d}, 1 \mathrm{H},{ }^{4} J=2.4 \mathrm{~Hz}, \mathrm{H}-(5)\right) ; 3.85$ (s, $\left.3 \mathrm{H},-\mathrm{OCH}_{3}\right)$; $2.93\left(\mathrm{ddd}, 1 \mathrm{H},{ }^{3} J=4.6 \mathrm{~Hz},{ }^{3} J=4.7 \mathrm{~Hz},{ }^{2} J=16.7 \mathrm{~Hz}, \mathrm{H}-(4)\right) ; 3.00$ (ddd, $1 \mathrm{H},{ }^{2} J=16.7 \mathrm{~Hz},{ }^{2} J=$ $\left.16.7 \mathrm{~Hz},{ }^{3} J=4.7 \mathrm{~Hz}, \mathrm{H}-(\mathrm{C} 4)\right) ; 2.54\left(\mathrm{ddq}, 1 \mathrm{H},{ }^{3} J=12.5 \mathrm{~Hz},{ }^{3} J=4.5 \mathrm{~Hz},{ }^{3} J=7.2 \mathrm{~Hz}, \mathrm{H}-(\mathrm{C} 2)\right)$; $2.16\left(\mathrm{dq}, 1 \mathrm{H},{ }^{3} J=4.5 \mathrm{~Hz},{ }^{3} J=9.0 \mathrm{~Hz},{ }^{2} J=13.0 \mathrm{~Hz}, \mathrm{H}-(\mathrm{C} 3)\right) ; 1.86\left(\mathrm{dddd}, 1 \mathrm{H},{ }^{3} J=5.1 \mathrm{~Hz},{ }^{2} J\right.$ $\left.=13.0 \mathrm{~Hz},{ }^{3} J=11.0 \mathrm{~Hz},{ }^{3} J=12.0 \mathrm{~Hz}, \mathrm{H}-(\mathrm{C} 3)\right) ; 1.25\left(\mathrm{~d}, 3 \mathrm{H}, J=7.0 \mathrm{~Hz}, \mathrm{C} 2-\mathrm{CH}_{3}\right)$.

${ }^{1} \mathrm{H}$ NMR spectral data are in agreement with those reported previously. ${ }^{15}$

2-Ethyl-3,4-dihydro-2H-naphthalen-1-one (4b). (2-Ethyl-1-tetralone). Reaction was carried out according to the „General procedure I“. After common work-up the desired product $\mathbf{4 b}$ was isolated as colorless oil in $65 \%$ yield.

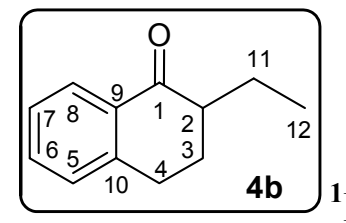

${ }^{1} \mathbf{H}$ NMR $\left(\mathrm{CDCl}_{3}, 300 \mathrm{MHz}, \delta\right): 8.03(\mathrm{dd}, 1 \mathrm{H}, J(7,8)=8.0, J(6,8)=1.5, \mathrm{H}-$ (C8)); $7.45(\mathrm{ddd}, 1 \mathrm{H}, J(5,6)=7.5, J(6,7)=7.5, J(6,8)=1.5$, H-(C6)); $7.29(\mathrm{dd}, 1 \mathrm{H}, J(7,8)=8.0$, $J(6,7)=7.5, \mathrm{H}-(\mathrm{C} 7)) ; 7.23$ (d, 1H, $J(5,6)=7.5, \mathrm{H}-(\mathrm{C} 5)) ; 3.03-2.95$ (m, 2H, H-(C4)); 2.46-2.35 (m, H, H-(C2)); 2.24 (ddd, $1 \mathrm{H},{ }^{2} J=13.5,{ }^{3} J=9.5,{ }^{3} J=4.5, \mathrm{H}-(\mathrm{C} 3)$ ); 2.05-1.83 (m, 2H, H-(C3, $\mathrm{C} 11)) ; 1.58\left(\mathrm{dq}, 1 \mathrm{H},{ }^{2} J=21.5, J(11,12)=7.5, \mathrm{H}-(\mathrm{C} 11)\right) ; 1.00(\mathrm{t}, 3 \mathrm{H}, J(11,12)=7.5, \mathrm{H}-(\mathrm{C} 12))$ ${ }^{13} \mathrm{C}$ NMR $\left(\mathrm{CDCl}_{3}, 75 \mathrm{MHz}, \delta\right): 200.46(\mathrm{C} 1), 144.18$ (C10), $133.23(\mathrm{CH}), 132.81$ (C9), 128.84 $(\mathrm{CH}), 127.62(\mathrm{CH}), 126.72(\mathrm{CH}), 49.12(\mathrm{C} 2), 28.58\left(\mathrm{CH}_{2}\right), 27.94\left(\mathrm{CH}_{2}\right), 22.63(\mathrm{C} 11), 11.64$ (C12). IR $\left(\mathrm{CHCl}_{3}, \mathrm{~cm}^{-1}\right): 2910$ (m, C-H Alif. $_{\text {. }}, 1669$ (s, C=O), 1592 (s, C=C), 1445 (s), 1424 (w), $1350(\mathrm{~m}), 1286(\mathrm{~m}), 1205$ (s), $1148(\mathrm{~m}), 1119(\mathrm{w}), 1086(\mathrm{w}), 1020(\mathrm{w}), 974(\mathrm{~m}), 898$ (s), $760(\mathrm{~s})$. Elemental analysis $\left(\mathrm{C}_{12} \mathrm{H}_{14} \mathrm{O}\right)$ : calc. $\mathrm{C} 82.72 \%$; H $8.10 \%$; found $\mathrm{C} 82.48 \%$; H $8.06 \%$. ${ }^{1} \mathrm{H}$ NMR and ${ }^{13} \mathrm{C}$ NMR spectral data are in agreement with those reported previously. ${ }^{15}$ 
2-Isopropyl-3,4-dihydronaphthalen-1(2H)-one (4c). (2-Isopropyl-1-tetralone). (a) Reaction was carried out according to the „General procedure I“. After common work-up the desired product $4 \mathbf{c}$ was isolated as colorless oil in $51 \%$ yield. Ecke gives b.p. $162^{\circ} \mathrm{C}(18 \mathrm{~mm})^{16}$.

(b) By basic hydrolysis and decarboxylation the yield was $86 \%$.

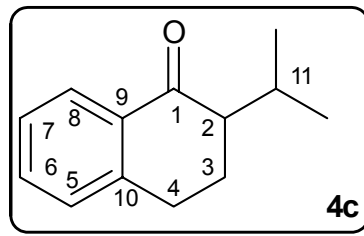

4c ${ }^{1} \mathbf{H}$ NMR $\left(300 \mathrm{MHz}, \mathrm{CDCl}_{3}, \delta\right): 8.03\left(\mathrm{dd}, 1 \mathrm{H},{ }^{3} \mathrm{~J}=7.8 \mathrm{~Hz},{ }^{4} J=1.2 \mathrm{~Hz}\right.$, $\mathrm{H}-(\mathrm{C} 8)) ; 7.45$ (dt, $\left.1 \mathrm{H},{ }^{3} J=7.5 \mathrm{~Hz},{ }^{4} J=1.2 \mathrm{~Hz}, \mathrm{H}-(\mathrm{C} 6)\right) ; 7.29$ (t, $\left.1 \mathrm{H},{ }^{3} J=8.1 \mathrm{~Hz}, \mathrm{H}-(\mathrm{C} 7)\right) ; 7.23$ (d, 1H, ${ }^{3} J=7.5 \mathrm{~Hz}, \mathrm{H}-(\mathrm{C} 5)$ ); 2.89-3.08 (m, 2H, H-(C3), H-(C4)); 2.53 (dsept., 1H, ${ }^{3} \mathrm{~J}=6.9 \mathrm{~Hz}$, $\left.{ }^{3} J=4.8 \mathrm{~Hz}, \mathrm{H}-(\mathrm{C} 11)\right) ; 2.33\left(\mathrm{ddd}, 1 \mathrm{H},{ }^{3} J=11.4 \mathrm{~Hz},{ }^{3} J=4.8 \mathrm{~Hz},{ }^{3} J=4.5 \mathrm{~Hz}, \mathrm{H}-(\mathrm{C} 4)\right) ; 2.11-2.20$ (m, 1H, H-(C2)); 1.95 (dddd, $1 \mathrm{H},{ }^{2} J=21 \mathrm{~Hz},{ }^{3} J=11.4 \mathrm{~Hz},{ }^{3} J=9.9 \mathrm{~Hz},{ }^{3} J=4.5 \mathrm{~Hz}, \mathrm{H}-(\mathrm{C} 3)$ ); $1.01\left(\mathrm{~d}, 3 \mathrm{H},{ }^{3} \mathrm{~J}=7.2 \mathrm{~Hz}, \mathrm{CH}_{3}-(i-P r)\right) ; 0.91\left(\mathrm{~d}, 3 \mathrm{H},{ }^{3} \mathrm{~J}=6.6 \mathrm{~Hz}, \mathrm{CH}_{3}-(i-P r)\right) .{ }^{13} \mathbf{C}$ NMR $(300$ $\left.\mathrm{MHz}, \mathrm{CDCl}_{3}, \delta\right): 200.10(\mathrm{C}=\mathrm{O}), 144.13\left(\mathrm{CH}_{\mathrm{Ar}}\right), 133.22\left(\mathrm{CH}_{\mathrm{Ar}}\right), 133.18\left(\mathrm{CH}_{\mathrm{Ar}}\right), 128.79\left(\mathrm{CH}_{\mathrm{Ar}}\right)$, $127.62\left(\mathrm{CH}_{\mathrm{Ar}}\right), 126.72\left(\mathrm{CH}_{\mathrm{Ar}}\right), 53.95(\mathrm{CH}(\mathrm{C} 2)), 28.76\left(\mathrm{CH}_{2}\right), 26.39(\mathrm{CH}-(i-P r)), 23.70\left(\mathrm{CH}_{2}\right)$, $20.89\left(\mathrm{CH}_{3}\right), 18.71\left(\mathrm{CH}_{3}\right)$. Elemental analysis $\left(\mathrm{C}_{13} \mathrm{H}_{16} \mathrm{O}\right)$ : calc. $\mathrm{C} 82.94 \%$; $\mathrm{H} 8.57 \%$; found $\mathrm{C}$ $83.84 \%$; $\mathrm{H} 9.27 \%$.

${ }^{1} \mathrm{H}$ NMR and ${ }^{13} \mathrm{C}$ NMR spectral data are in agreement with those reported previously. ${ }^{17}$

2-Allyl-3,4-dihydronaphthalen-1(2H)-one (4d). (2-Allyl-1-tetralone). Reaction was carried out according to the „General procedure II“. After common work-up the desired product 4d was isolated as colorless oil in $87 \%$ yield.

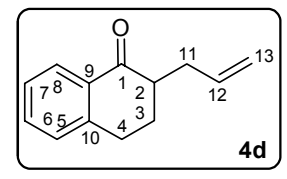

${ }^{1} \mathbf{H}$ NMR $\left(300 \mathrm{MHz}, \mathrm{CDCl}_{3}, \delta\right): 8.04\left(\mathrm{dd}, 1 \mathrm{H},{ }^{3} \mathrm{~J}=7.8 \mathrm{~Hz},{ }^{4} \mathrm{~J}=1.2 \mathrm{~Hz}, \mathrm{H}-(\mathrm{C} 8)\right)$; $7.46\left(\mathrm{dt}, 1 \mathrm{H},{ }^{3} J=7.5 \mathrm{~Hz},{ }^{4} J=1.2 \mathrm{~Hz}, \mathrm{H}-(\mathrm{C} 6)\right) ; 7.30$ (t, $\left.1 \mathrm{H},{ }^{3} J=7.2 \mathrm{~Hz}, \mathrm{H}-(\mathrm{C} 7)\right) ; 7.23$ (d, $1 \mathrm{H},{ }^{3} J$ $=7.5 \mathrm{~Hz}, \mathrm{H}-(\mathrm{C} 5)) ; 5.78-5.92(\mathrm{~m}, 1 \mathrm{H}, \mathrm{H}-(\mathrm{C} 12)) ; 5.05-5.14(\mathrm{~m}, 2 \mathrm{H}, 2 \mathrm{xH}-(\mathrm{C} 13)) ; 2.98-3.02(\mathrm{~m}$, 2H, 2xH-(C4)); 2.72-2.81 (m, 1H, H-(C2)); 2.50-2.60 (m, 1H, H-(C11)); 2.20-2.32 (m, 2H, H(C3), H-(C11)); 1.80-1.94 (m, 1H, H-(C3)). $\left.{ }^{13} \mathbf{C ~ N M R ~ ( 3 0 0 ~ M H z , ~} \mathrm{CDCl}_{3}, \delta\right): 199.65(\mathrm{C}=\mathrm{O})$, $144.26(\mathrm{C}), 136.43\left(\mathrm{CH}_{\mathrm{Ar}}\right), 133.40\left(\mathrm{CH}_{\mathrm{Ar}}\right), 132.73(\mathrm{C}), 128.91\left(\mathrm{CH}_{\mathrm{Ar}}\right), 127.68\left(\mathrm{CH}_{\mathrm{Ar}}\right), 126.79$ (C13), $117.03(\mathrm{C} 12), 47.40(\mathrm{C} 2), 34.26\left(\mathrm{CH}_{2}\right), 28.83\left(\mathrm{CH}_{2}\right), 28.18\left(\mathrm{CH}_{2}\right)$. Elemental analysis $\left(\mathrm{C}_{13} \mathrm{H}_{14} \mathrm{O}\right)$ : calc. C 83.83\%; $\mathrm{H} 7.58 \%$; found $\mathrm{C} 82.73 \%$; $\mathrm{H} 7.50 \%$.

2- Benzyl -3,4-dihydronaphthalen-1(2H)-one (4e). (2-Benzyl-1-tetralone). Reaction was carried out according to the "General procedure II“. After common work-up and column chromatography $\left(\mathrm{SiO}_{2}\right.$, Hexsol:EtOAc - 5:1 to 5:2) desired product 4e was isolated as yellowish oil in $93 \%$ yield. 


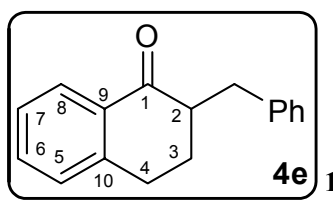

${ }^{1} \mathbf{H}-\mathbf{N M R}\left(300 \mathrm{MHz}, \mathrm{CDCl}_{3}, \delta\right): 8.07\left(\mathrm{dd}, 1 \mathrm{H},{ }^{4} J(8,6)=1.2,{ }^{3} J(8,7)=7.8 \mathrm{~Hz}\right.$, $\mathrm{H}-(\mathrm{C} 8)) ; 7.46\left(\mathrm{ddd},{ }^{4} J(6,8)=1.3,{ }^{3} J(6,5)={ }^{3} J(6,7)=7.5 \mathrm{~Hz}, \mathrm{H}-(\mathrm{C} 6)\right) ; 7.20-7.35$ (m, 7H, H-(C5) $+\mathrm{H}-(\mathrm{C} 7)+5 \mathrm{xH}-(\mathrm{Ph})) ; 3.50\left(\mathrm{dd}, 1 \mathrm{H},{ }^{3} J\left(2, \mathrm{PhCH}_{2^{-}}\right)={ }^{3} J(2,3)=4.5,{ }^{3} J\left(2,-\mathrm{CH}_{2}-\mathrm{Ph}\right)={ }^{3} J(2,3)=\right.$ $13.4 \mathrm{~Hz}, \mathrm{H}-(\mathrm{C} 2)) ; 2.93\left(\mathrm{dd}, 1 \mathrm{H},{ }^{3} J\left(-\mathrm{CH}_{2}-\mathrm{Ph}, 2\right)=4.5,{ }^{2} \mathrm{~J}=10.0 \mathrm{~Hz},-\mathrm{CH}_{2}-\mathrm{Ph}\right) ; 2.70-2.95(\mathrm{~m}, 2 \mathrm{H}$, $2 \mathrm{xH}-(\mathrm{C} 4)) ; 2.64\left(\mathrm{dd}, 1 \mathrm{H},{ }^{2} J=10.0,{ }^{3} J\left(-\mathrm{CH}_{2}-\mathrm{Ph}, 2\right)=13.4 \mathrm{~Hz},-\mathrm{CH}_{2}-\mathrm{Ph}\right) ; 2.12$ (m, 1H, with measurable $\left.{ }^{3} J(3,2)=4.3,{ }^{2} J=11.5 \mathrm{~Hz}, \mathrm{H}-(\mathrm{C} 3)\right) ; 1.79$ [dddd, $1 \mathrm{H},{ }^{3} J(3,4)=6.0,{ }^{3} J(3,4)=9.6 \mathrm{~Hz}$, $\left.{ }^{2} J=11.5,{ }^{3} J(3,2)=13.4 \mathrm{~Hz}, \mathrm{H}-(\mathrm{C} 3)\right)$. IR $\left(\mathrm{CH}_{2} \mathrm{Cl}_{2}, \mathrm{~cm}^{-1}\right): 2913\left(\mathrm{~m}, \mathrm{C}-\mathrm{H}_{\text {alif. }}\right), 1679$ (s, C=O), 1269 (m), 1223 (m), 737 (s, C-Harom.), 706 (s, C-Harom.).

${ }^{1} \mathrm{H}$ NMR spectral data are in agreement with those reported previously. ${ }^{5}$

Preparation of 2-hydroxy-2-alkyl-3,4-dihydronaphthalen-1(2H)-ones (5-5e). (2-hydroxy-2alkyl-1-tetralones)

General procedure. The corresponding 2-alkyl-1-tetralone (4-4e) (2.37 mmol, 1.0 equiv.) was dissolved in toluene $(15 \mathrm{~mL})$ covered with freshly prepared $50 \%$ solution of $\mathrm{NaOH}(10 \mathrm{~mL})$. Triethylbenzylammonium chloride (TEBA) (0.36 mmol, 0.15 equiv.) and trimethoxy phosphite $\left(\mathrm{P}(\mathrm{OMe})_{3}\right),(2.96 \mathrm{mmol}, 1.25$ equiv.) were successively added. Reaction mixture was vigorously stirred at rt and oxygen (or air via small pumping device) was bubbled via a syringe through the mixture. After 4 - $24 \mathrm{~h}$ starting material was not detected (TLC control). Solution was diluted with water $(30 \mathrm{~mL})$ and EtOAc $(20 \mathrm{~mL})$. Aqueous layer was extracted with EtOAc $(3 \mathrm{x} 20 \mathrm{~mL})$. Combined organic layers were washed with $10 \% \mathrm{HCl}(30 \mathrm{~mL})$, water $(30 \mathrm{~mL})$ and dried over $\mathrm{Na}_{2} \mathrm{SO}_{4}$. After filtration and removal of the solvent, the crude product was isolated and purified.

2-Hydroxy-2-methyl-3,4-dihydronaphthalen-1(2H)-one (5). (2-Hydroxy-2-methyl-1tetralone). Crude product was distilled at reduced pressure $\left(85-90^{\circ} \mathrm{C} / 8 \mathrm{~Pa}\right)$. Desired 2-hydroxy2-methyltetralone (5) was isolated as yellowish oil in $95 \%$ yield.

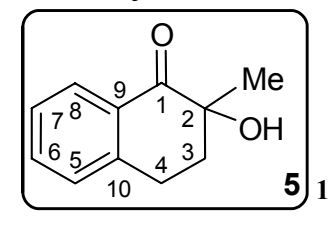

${ }^{1} \mathbf{H}$ NMR $\left(\mathrm{CDCl}_{3}, 300 \mathrm{MHz}, \delta\right): 8.05(\mathrm{dd}, 1 \mathrm{H}, J(7,8)=8.0, J(6,8)=1.5, \mathrm{H}-$ $(\mathrm{C} 8)) ; 7.53(\mathrm{ddd}, 1 \mathrm{H}, J(5,6)=7.5, J(6,7)=7.5, J(6,8)=1.5, \mathrm{H}-(\mathrm{C} 6)) ; 7.35(\mathrm{dd}, 1 \mathrm{H}, J(7,8)=8.0$, $J(6,7)=7.5, \mathrm{H}-(\mathrm{C} 7)) ; 7.27(\mathrm{~d}, 1 \mathrm{H}, J(5,6)=7.5, \mathrm{H}-(\mathrm{C} 5)) ; 3.86(\mathrm{bs}, 1 \mathrm{H}, \mathrm{HO}-(\mathrm{C} 2)) ; 3.12(\mathrm{ddd}, 1 \mathrm{H}$, $\left.{ }^{2} J=17.5, J\left(3^{\mathrm{Me}-\mathrm{C}(2)}, 4^{\mathrm{OH}}\right)=11.0, J\left(3^{\mathrm{OH}}, 4^{\mathrm{OH}}\right)=6.0, \mathrm{H}^{\mathrm{OH}}-(\mathrm{C} 4)\right) ; 3.02\left(\mathrm{ddd}, 1 \mathrm{H},{ }^{2} J=17.5, J\left(3^{\mathrm{Me}-}\right.\right.$ $\left.\left.{ }^{\mathrm{C}(2)}, 4^{\mathrm{Me}-\mathrm{C}(2)}\right)=5.5, J\left(3^{\mathrm{C}(2)}, 4^{\mathrm{Me}-\mathrm{C}(2)}\right)=3.5, \mathrm{H}^{\mathrm{Me}-\mathrm{C}(2)}-(\mathrm{C} 4)\right) ; 2.28\left(\mathrm{ddd}, 1 \mathrm{H},{ }^{2} J=13.0, J\left(3^{\mathrm{OH}}, 4^{\mathrm{OH}}\right)=\right.$ $\left.6.0, J\left(3^{\mathrm{OH}}, 4^{\mathrm{Me}-\mathrm{C}(2)}\right)=3.5, \mathrm{H}^{\mathrm{OH}}-(\mathrm{C} 3)\right) ; 2.22\left(\mathrm{ddd}, 1 \mathrm{H},{ }^{2} J=13.0, J\left(3^{\mathrm{Me}-\mathrm{C}(2)}, 4^{\mathrm{OH}}\right)=11.0, J\left(3^{\mathrm{Me}-}\right.\right.$ $\left.\left.{ }^{\mathrm{C}(2)}, 4^{\mathrm{Me}-\mathrm{C}(2)}\right)=5.5, \mathrm{H}^{\mathrm{Me}-\mathrm{C}(2)}-(\mathrm{C} 3)\right) ; 1.40(\mathrm{~s}, 3 \mathrm{H}, \mathrm{Me}-(\mathrm{C} 2)) .{ }^{\mathbf{1 3}} \mathbf{C ~ N M R}\left(\mathrm{CDCl}_{3}, 75 \mathrm{MHz}, \delta\right): 201.82$ (C1), 143.42 (C10), $134.08(\mathrm{CH}), 129.92$ (C9), $129.01(\mathrm{CH}), 128.03(\mathrm{CH}), 126.91(\mathrm{CH}), 73.60$ 
(C2), 35.88 (C3), 26.83 (C4), 23.91 (Me-(C2)). ${ }^{1} \mathrm{H}$ NMR and ${ }^{13} \mathrm{C}$ NMR spectral data are in agreement with those reported previously. ${ }^{2}$

2-Hydroxy--6-methoxy-2-methyl-3,4-dihydronaphthalen-1(2H)-one (5a). (2-Hydroxy-6methoxy-2-methyl-1-tetralone). Product 5a was prepared in 49\% yield like light yellow crystalline compound, with m.p. $74-76^{\circ} \mathrm{C}$.

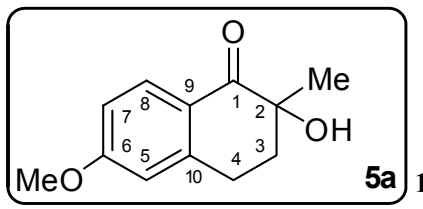

$\left(\mathrm{dd}, 1 \mathrm{H},{ }^{4} \mathrm{~J}=2.4 \mathrm{~Hz},{ }^{3} \mathrm{~J}=8.7 \mathrm{~Hz}, \mathrm{H}-(\mathrm{C} 7)\right) ; 6.69$ (d, $\left.1 \mathrm{H},{ }^{4} \mathrm{~J}=2.1 \mathrm{~Hz}, \mathrm{H}-(\mathrm{C} 5)\right) ; 3.89$ (bs, $\left.1 \mathrm{H},-\mathrm{OH}\right)$; 3.87 (s, 3H, $\left.-\mathrm{OCH}_{3}\right) ; 3.09$ (ddd, $1 \mathrm{H},{ }^{3} \mathrm{~J}=6.0 \mathrm{~Hz},{ }^{3} J=11.0 \mathrm{~Hz},{ }^{2} J=17.4 \mathrm{~Hz}, \mathrm{H}-(\mathrm{C} 4)$ ); 2.98 (ddd, $\left.1 \mathrm{H},{ }^{3} J=5.4 \mathrm{~Hz},{ }^{2} J=17.7 \mathrm{~Hz},{ }^{3} J=2.7 \mathrm{~Hz}, \mathrm{H}-(\mathrm{C} 4)\right) ;, 2.24\left(\mathrm{ddd}, 1 \mathrm{H},{ }^{3} J=3.0 \mathrm{~Hz},{ }^{2} J=13.2 \mathrm{~Hz},{ }^{3} J\right.$ $=6.0 \mathrm{~Hz}, \mathrm{H}-(\mathrm{C} 3)) ; 2.20\left(\mathrm{ddd}, 1 \mathrm{H},{ }^{3} J=5.4 \mathrm{~Hz},{ }^{3} \mathrm{~J}=11.0 \mathrm{~Hz},{ }^{2} J=13.0 \mathrm{~Hz}, \mathrm{H}-(\mathrm{C} 3)\right) ; 1.38(\mathrm{~s}, 3 \mathrm{H}$, $\left.(\mathrm{C} 2)-\mathrm{CH}_{3}\right) ; .{ }^{13} \mathbf{C}$ NMR $\left(\mathrm{CDCl}_{3}, 75 \mathrm{MHz}, \delta\right): 200.63$ (C1), 164.41 (C6), $146.21\left(\mathrm{C}_{\mathrm{Ar}}\right), 130.67$ $(\mathrm{CH}), 123.48\left(\mathrm{C}_{\mathrm{Ar}}\right), 113.95(\mathrm{CH}), 112.89(\mathrm{CH}), 73.49(\mathrm{C} 2), 55.71(-\mathrm{OMe}), 36.09(\mathrm{C} 3), 27.41$ (C4), $24.40(\mathrm{Me}-(\mathrm{C} 2))$. IR $\left(\mathrm{CHCl}_{3} \cdot \mathrm{cm}^{1-}\right): 3400$ (w, O-H); $3000\left(\mathrm{~m}, \mathrm{C}_{\mathrm{alk}}-\mathrm{H}\right) ; 1660$ (s, C=O); 1250 $\left(-\mathrm{OCH}_{3}\right)$. Elemental analysis $\left(\mathrm{C}_{12} \mathrm{H}_{14} \mathrm{O}_{3}\right)$ : calc. $\mathrm{C} 69.89 \% ; \mathrm{H} 6.84 \%$; found $\mathrm{C} 69.96 \% ; \mathrm{H} 6.95 \%$.

\section{2-Ethyl-2-hydroxy-3,4-dihydronaphthalen-1(2H)-one (5b). (2-Ethyl-2-hydroxy-1-tetralone)} Product $\mathbf{5 b}$ was isolated in $78 \%$ yield like colorless oil.

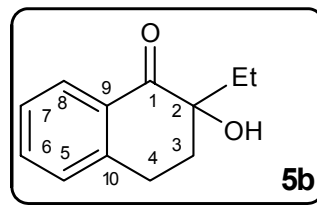

$(\mathrm{C} 8)) ; 7.52(\mathrm{ddd}, 1 \mathrm{H}, J(6,7)=8.0, J(5,6)=7.5, J(6,8)=1.5, \mathrm{H}-(\mathrm{C} 6)) ; 7.34(\mathrm{dd}, 1 \mathrm{H}, J(6,7)=8.0$, $J(7,8)=7.5, \mathrm{H}-(\mathrm{C} 7)) ; 7.25(\mathrm{~d}, 1 \mathrm{H}, J(5,6)=7.5, \mathrm{H}-(\mathrm{C} 5)) ; 3.82$ (s, 1H, HO-(C2)); 3.10 (ddd, 1H, $\left.{ }^{2} J=17.5, J\left(3^{\mathrm{Et}-\mathrm{C}(2)}, 4^{\mathrm{OH}}\right)=13.0, J\left(3^{\mathrm{OH}}, 4^{\mathrm{OH}}\right)=5.0, \mathrm{H}^{\mathrm{OH}}-(\mathrm{C} 4)\right) ; 2.99\left(\mathrm{ddd}, 1 \mathrm{H},{ }^{2} J=17.5, J\left(3^{\mathrm{Et}-}\right.\right.$ $\left.\left.{ }^{\mathrm{C}(2)}, 4^{\mathrm{Et}-\mathrm{C}(2)}\right)=6.0, J\left(3^{\mathrm{OH}}, 4^{\mathrm{Et}-\mathrm{C}(2)}\right)=2.5, \mathrm{H}^{\mathrm{Et}-\mathrm{C}(2)}-(\mathrm{C} 4)\right) ; 2.34\left(\mathrm{ddd}, 1 \mathrm{H},{ }^{2} J=13.5, J\left(3^{\mathrm{OH}}, 4^{\mathrm{OH}}\right)=5.0\right.$, $\left.J\left(3^{\mathrm{OH}}, 4^{\mathrm{Et}-\mathrm{C}(2)}\right)=2.5, \mathrm{H}^{\mathrm{OH}}-(\mathrm{C} 3)\right) ; 2.16\left(\mathrm{ddd}, 1 \mathrm{H},{ }^{2} J=13.5, J\left(3^{\mathrm{Et}-\mathrm{C}(2)}, 4^{\mathrm{OH}}\right)=13.0, J\left(3^{\mathrm{Et}-\mathrm{C}(2)}, 4^{\mathrm{Et}-\mathrm{C}(2)}\right)=\right.$ 6.0, $\left.\mathrm{H}^{\mathrm{Et}-\mathrm{C}(2)}-(\mathrm{C} 3)\right) ; 1.73\left(\mathrm{dq}, 1 \mathrm{H},{ }^{2} J=14.5, J(11,12)=7.5, \mathrm{H}-(\mathrm{C} 11)\right) ; 1.65\left(\mathrm{dq}, 1 \mathrm{H},{ }^{2} J=14.5\right.$, $J(11,12)=7.5, \mathrm{H}-(\mathrm{C} 11)) ; 0.94(\mathrm{t}, 3 \mathrm{H}, J(11,12)=7.5, \mathrm{H}-(\mathrm{C} 12)) .{ }^{13} \mathbf{C} \mathbf{N M R}\left(\mathrm{CDCl}_{3}, 75 \mathrm{MHz}, \delta\right)$ : 202.15 (C1), 143.62 (C10), $134.14(\mathrm{CH}), 130.47$ (C9), $129.17(\mathrm{CH}), 128.07(\mathrm{CH}), 127.05(\mathrm{CH})$, 76.00 (C2), 33.77 (C3), 28.57 (C11), 26.69 (C4), 7.38 (C12). ${ }^{1} \mathrm{H}$ NMR and ${ }^{13} \mathrm{C}$ NMR spectral data are in agreement with those reported previously. ${ }^{11}$

2-Isopropyl-2-hydroxy-3,4-dihydronaphthalen-1(2H)-one (5c). (2-Isopropyl-2-hydroxy-1tetralone). Product 5c was prepared in 50\% yield like white crystalline compound, with m.p. 59$62^{\circ} \mathrm{C}\left(\mathrm{Et}_{2} \mathrm{O}\right)$. Carnduff ${ }^{18}$ refers m.p. $66-68^{\circ} \mathrm{C}$ (from petroleum). 


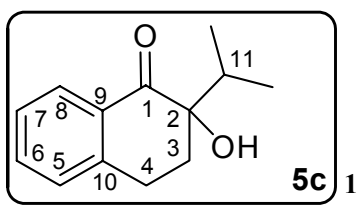

${ }^{1} \mathbf{H}$ NMR $\left(300 \mathrm{MHz}, \mathrm{CDCl}_{3}, \delta\right): 7.97\left(\mathrm{dd}, 1 \mathrm{H},{ }^{3} J=7.5 \mathrm{~Hz},{ }^{4} J=1.5 \mathrm{~Hz}, \mathrm{H}-\right.$ (C8)); $7.51\left(\mathrm{dt}, 1 \mathrm{H},{ }^{3} J=7.5 \mathrm{~Hz},{ }^{4} J=1.5 \mathrm{~Hz}, \mathrm{H}-(\mathrm{C} 6)\right) ; 7.33\left(\mathrm{t}, 1 \mathrm{H},{ }^{3} J=7.5 \mathrm{~Hz}, \mathrm{H}-(\mathrm{C} 7)\right) ; 7.24$ (d, $\left.1 \mathrm{H},{ }^{3} \mathrm{~J}=8.1 \mathrm{~Hz}, \mathrm{H}-(\mathrm{C} 5)\right) ; 3.76(\mathrm{~s}, 1 \mathrm{H},-\mathrm{OH}) ; 3.10\left(\mathrm{ddd}, 1 \mathrm{H},{ }^{2} \mathrm{~J}=17.7 \mathrm{~Hz},{ }^{3} \mathrm{~J}=12.6 \mathrm{~Hz},{ }^{3} \mathrm{~J}=4.8\right.$ Hz, H-(C4)); 2.97 (ddd, 1H, ${ }^{2} J=17.7 \mathrm{~Hz},{ }^{3} J=5.7 \mathrm{~Hz},{ }^{3} J=2.1 \mathrm{~Hz}, \mathrm{H}-(\mathrm{C} 4)$ ); 2.46 (ddd, $1 \mathrm{H},{ }^{2} J=$ $\left.14.1 \mathrm{~Hz},{ }^{3} J=4.8 \mathrm{~Hz},{ }^{3} \mathrm{~J}=2.1 \mathrm{~Hz}, \mathrm{H}-(\mathrm{C} 3)\right) ; 2.08\left(\mathrm{ddd}, 1 \mathrm{H},{ }^{2} J=18.6 \mathrm{~Hz},{ }^{3} \mathrm{~J}=12.6 \mathrm{~Hz},{ }^{3} J=5.7\right.$ $\mathrm{Hz}, \mathrm{H}-(\mathrm{C} 3)$ ); 2.01 (sept., $1 \mathrm{H},{ }^{3} \mathrm{~J}=6.6 \mathrm{~Hz}, \mathrm{H}-(\mathrm{C} 11)$ ); 1.08 (d, 3H, ${ }^{3} \mathrm{~J}=6.6 \mathrm{~Hz}, \mathrm{CH}_{3}-(\mathrm{i}-\mathrm{Pr})$ ); 0.73 $\left(\mathrm{d}, 3 \mathrm{H},{ }^{3} \mathrm{~J}=6.6 \mathrm{~Hz}, \mathrm{CH}_{3}-(\mathrm{i}-\mathrm{Pr})\right) .{ }^{13} \mathrm{C}$ NMR $\left(300 \mathrm{MHz}, \mathrm{CDCl}_{3}, \delta\right): 202.87(\mathrm{C}=\mathrm{O}), 143.64(\mathrm{C})$, $133.99\left(\mathrm{CH}_{\mathrm{Ar}}\right), 130.99(\mathrm{C}), 129.11\left(\mathrm{CH}_{\mathrm{Ar}}\right), 128.03\left(\mathrm{CH}_{\mathrm{Ar}}\right), 127.04\left(\mathrm{CH}_{\mathrm{Ar}}\right), 77.63(\mathrm{C} 2), 32.86$ $\left(\mathrm{CH}_{2}\right), 29.63(\mathrm{CH}), 26.42\left(\mathrm{CH}_{2}\right), 16.44\left(\mathrm{CH}_{3}\right), 16.18\left(\mathrm{CH}_{3}\right) . \mathbf{I R}\left(\mathrm{CHCl}_{3}, \mathrm{~cm}^{-1}\right) 3500(\mathrm{w}, \mathrm{O}-\mathrm{H})$, $2880\left(\mathrm{~s}, \mathrm{C}_{\mathrm{Ar}}-\mathrm{H}\right), 1695$ (s, C=O), 1625 (w, C=C), $1480(\mathrm{~m}), 1380$ (m), 1275 (s, C-O), 995 (m), 445 (s). Elemental analysis $\left(\mathrm{C}_{13} \mathrm{H}_{16} \mathrm{O}_{2}\right)$ : calc. $\mathrm{C} 76.44 \%$; $\mathrm{H} .90 \%$; found $\mathrm{C} 76.87 \% ; \mathrm{H} \mathrm{8.06 \%}$. Infrared and ${ }^{1} \mathrm{H}$ NMR spectral data are in agreement with those reported previously. ${ }^{18}$

2-Allyl-2-hydroxy-3,4-dihydronaphthalen-1(2H)-one (5d). (2-Allyl-2-hydroxy-1-tetralone). Product 5d was isolated after purification by chromatography $\left(\mathrm{SiO}_{2}, \mathrm{Hexsol}: \mathrm{Et}_{2} \mathrm{O}-5: 1\right)$ in $95 \%$ yield like yellowish oil.

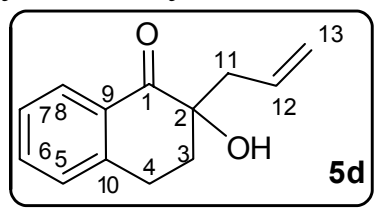

5d ${ }^{1} \mathbf{H}$ NMR $\left(300 \mathrm{MHz}, \mathrm{CDCl}_{3}, \delta\right): 8.02\left(\mathrm{dd}, 1 \mathrm{H},{ }^{3} \mathrm{~J}=7.8 \mathrm{~Hz},{ }^{4} \mathrm{~J}=1.5 \mathrm{~Hz}, \mathrm{H}-\right.$ (C8)); $7.53\left(\mathrm{dt}, 1 \mathrm{H},{ }^{3} J=7.8 \mathrm{~Hz},{ }^{4} J=1.5 \mathrm{~Hz}, \mathrm{H}-(\mathrm{C} 6)\right) ; 7.35$ (t, $\left.1 \mathrm{H},{ }^{3} J=7.8 \mathrm{~Hz}, \mathrm{H}-(\mathrm{C} 7)\right) ; 7.26$ (d, $\left.1 \mathrm{H},{ }^{3} \mathrm{~J}=7.8 \mathrm{~Hz}, \mathrm{H}-(\mathrm{C} 5)\right) ; 5.88\left(\mathrm{dddd}, 1 \mathrm{H},{ }^{3} \mathrm{~J}=23.1 \mathrm{~Hz},{ }^{3} \mathrm{~J}=14.1 \mathrm{~Hz},{ }^{3} \mathrm{~J}=7.8 \mathrm{~Hz},{ }^{3} \mathrm{~J}=6.3 \mathrm{~Hz}\right.$, $\mathrm{H}-(\mathrm{C} 12))$; 5.05-5.19 (m, 2H, 2xH-(C13)); 3.81 (s, 1H, -OH); 3.11 (ddd, $1 \mathrm{H},{ }^{2} J=17.7 \mathrm{~Hz},{ }^{3} J=$ $\left.12.6 \mathrm{~Hz},{ }^{3} \mathrm{~J}=5.1 \mathrm{~Hz}, \mathrm{H}-(\mathrm{C} 4)\right) ; 2.99\left(\mathrm{ddd}, 1 \mathrm{H},{ }^{2} \mathrm{~J}=17.7 \mathrm{~Hz},{ }^{3} \mathrm{~J}=6 \mathrm{~Hz},{ }^{3} J=2.4 \mathrm{~Hz}, \mathrm{H}-(\mathrm{C} 4)\right)$; $2.32-$ $2.48(\mathrm{~m}, 3 \mathrm{H}, 2 \mathrm{xH}-(\mathrm{C} 11)+\mathrm{H}-(\mathrm{C} 3)) ; 2.16\left(\mathrm{ddd}, 1 \mathrm{H},{ }^{2} J=18.9 \mathrm{~Hz},{ }^{3} J=12.9 \mathrm{~Hz},{ }^{3} J=6 \mathrm{~Hz}, \mathrm{H}-\right.$ (C3)). ${ }^{13} \mathbf{C}$ NMR (300 MHz, $\left.\mathrm{CDCl}_{3}, \delta\right): 201.25(\mathrm{C}=\mathrm{O}), 143.62(\mathrm{C}), 134.30\left(\mathrm{CH}_{\mathrm{Ar}}\right), 132.27$ $\left(\mathrm{CH}_{\mathrm{Ar}}\right), 130.29(\mathrm{C}), 129.25\left(\mathrm{CH}_{\mathrm{Ar}}\right), 128.18\left(\mathrm{CH}_{\mathrm{Ar}}\right), 127.14(\mathrm{C} 12), 119.38(\mathrm{C} 13), 75.57(\mathrm{C} 2)$, $40.51\left(\mathrm{CH}_{2}\right), 33.68\left(\mathrm{CH}_{2}\right), 26.36\left(\mathrm{CH}_{2}\right)$. Elemental analysis $\left(\mathrm{C}_{13} \mathrm{H}_{14} \mathrm{O}_{2}\right)$ : calc. $\mathrm{C} 77.20 \% ; \mathrm{H}$ $6.98 \%$; found C $76.08 \%$; H $6.99 \%$.

${ }^{1} \mathrm{H}$ NMR and ${ }^{13} \mathrm{C}$ NMR spectral data are in agreement with those reported previously. ${ }^{11}$

2-Benzyl-2-hydroxy-3,4-dihydronaphthalen-1(2H)-one (5e). (2-Benzyl-2-hydroxy-1tetralone). Product 5e was prepared in $89 \%$ yield like yellowish crystalline compound, m.p. $104.0-105.1{ }^{\circ} \mathrm{C}\left(\mathrm{Hexsol} / \mathrm{Et}_{2} \mathrm{O}\right)$. 


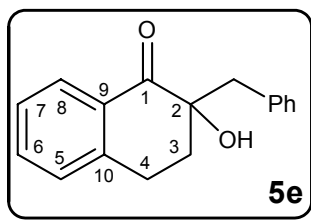

${ }^{1} \mathbf{H}-\mathbf{N M R}\left(300 \mathrm{MHz}, \mathrm{CDCl}_{3}, \delta\right): 8.02\left(\mathrm{dd}, 1 \mathrm{H},{ }^{4} J(8,6)=1.3,{ }^{3} J(8,7)=7.8 \mathrm{~Hz}\right.$, $\mathrm{H}-(\mathrm{C} 8)) ; 7.57\left(\mathrm{ddd}, 1 \mathrm{H},{ }^{4} J(6,8)=1.3,{ }^{3} J(6,5)={ }^{3} J(6,7)=7.5 \mathrm{~Hz}, \mathrm{H}-(\mathrm{C} 6)\right) ; 7.13-7.41$ (m, 7H, H$(\mathrm{C} 5)+\mathrm{H}-(\mathrm{C} 7)+5 \mathrm{x} \mathrm{H}-(\mathrm{Ph})) ; 3.76(\mathrm{bs}, 1 \mathrm{H},-\mathrm{OH}) ; 3.27\left(\mathrm{ddd}, 1 \mathrm{H},{ }^{3} J(4,3)=5.7,{ }^{3} J(4,3)=12.2,{ }^{2} J\right.$ $=18.0 \mathrm{~Hz}, \mathrm{H}-(\mathrm{C} 4)) ; 3.04\left(\mathrm{ddd}, 1 \mathrm{H},{ }^{3} J(4,3)=2.4,{ }^{3} J(4,3)=5.4,{ }^{2} J=18.0 \mathrm{~Hz}, \mathrm{H}-(\mathrm{C} 4)\right) ; 2.92(\mathrm{~d}$, $\left.1 \mathrm{H},{ }^{2} \mathrm{~J}=13.8 \mathrm{~Hz},-\mathrm{CH}_{2} \mathrm{Ph}\right) ; 3.00\left(\mathrm{~d}, 1 \mathrm{H},{ }^{2} \mathrm{~J}=13.8 \mathrm{~Hz},-\mathrm{CH}_{2} \mathrm{Ph}\right) ; 2.20-2.32$ (m, 1H, with measurable $\left.{ }^{3} J(3,4)=2.4,{ }^{3} J(3,4)=5.7, J=7.8 \mathrm{~Hz}, \mathrm{H}-(\mathrm{C} 3)\right) ; 2.14-2.28$ (m, 1H, with measurable $\left.{ }^{3} J(3,4)=5.4,{ }^{3} J(3,4)=12.2 \mathrm{~Hz}, \mathrm{H}-(\mathrm{C} 3)\right) .{ }^{13} \mathbf{C}-\mathbf{N M R}\left(75 \mathrm{MHz}, \mathrm{CDCl}_{3}, \delta\right): 200.87(\mathrm{C} 1, \mathrm{C}=\mathrm{O})$, 143.18 (C10-Ar), 135.32 (C9-Ar), 134.12 (CHAr), 130.39 (C-Ar, Ph), 130.35 (2 x CHAr), 129.10 (CHAr), 128.09 (2 x CHAr), 128.00 (CHAr), 127.06 (CHAr), 126.86 (CHAr), 76.04 (C2), 41.95 (-CH2 $-\mathrm{Ph}), 33.82(\mathrm{C} 3), 26.38(\mathrm{C} 4) . \mathbf{I R}\left(\mathrm{CH}_{2} \mathrm{Cl}_{2}, \mathrm{~cm}^{-1}\right): 3315$ (s, -OH), 3010 (m, C$\mathrm{H}_{\text {alif. }}$ ), 1679 (s, C=O), 1277 (s, C-O, doublet), 760 (s, C-H arom.), 706 (s, C-H arom.).

\section{Acknowledgements}

This work was supported by the Slovak Research and Development Agency under the contract No. APVT-20-031904, APVV LPP 0153-06 and VEGA 1/4467/07.

\section{References and Notes}

1. (a) Solladié-Cavallo, A.; Šedý, O.; Sališová, M.; Schmitt, M. Eur. J. Org. Chem. 2002, 3042. See also supporting information. (b) Pažický, M.; Gášpár, B.; Solladié-Cavallo, A.; Sališová, M.; Boháč, A; Hutta, M.; Addová, G. Synthesis 2006, 2013.

2. Solladié-Cavallo, A.; Šedý, O.; Sališová, M.; Biba, M.; Welch, J.; Nafié, L.; Freedman, T. Tetrahedron: Asymmetry 2001, 12, 2703; and references therein.

3. (a) Davis, F. A.; Weismiller, M. C. J. Org. Chem. 1990, 55, 3715. (b) Takikawa, H.; Hachisu, Y.; Bode, J. W.; Suzuki, K. Angew. Chem. Int. Ed. 2006, 45, 3492. (c) Masui, M.; Ando, A.; Shioiri, T. Tetrahedron Lett. 1988, 29, 2835.

4. (a) Davis, F. A.; Zhou, P.; Murphy, C. K.; Sundarababu, G.; Qi, H.; Han, W.; Przeslawski, R.M.; Chen, B.-C.; Carroll, P. J. J. Org. Chem. 1998, 63, 2273. (b) Murphy, J. A.; Mahesh, M.; McPheators, G.; Anand, R. V.; McGuire, T. M.; Carling, R.; Kennedy, A. R. Org. Lett. 2007, 9, 3233.

5. (a) Streitwieser, A.; Kim, Y.-J.; Wang, D. Z.-R. Org. Lett. 2001, 3, 2599. See also supporting information. (b) Goto, M.; Akimoto, K.-I.; Aoki, K.; Shindo, M.; Koga, K Tetrahedron Lett. 1999, 40, 8129. (c) Adamczyk, M.; Watt, D. S.; Netzel, D. A. J. Org. Chem. 1984, 49, 4226. 
6. Semak, V.; Boháč, A.; Sališová, M.; Addová, G.; Danko, P. Chem. Papers 2008, 62, 275.

7. Loewenthal, H. J. E. Guide for the Perplexed Organic Experimentalist; Heyden: London, 1980; pp 125-128.

8. Brown D. S.; Marples, B. A.; Smith, P.; Walton, L. Tetrahedron 1995, 51, 3587.

9. Park, E. L; Kim, M. H; Kim, D. Y. J. Org. Chem. 2004, 69, 6897 and references therein.

10. Krapcho, A. P. Synthesis 1982, 805 and 893.

11. de Vries, E. F. J.; Ploeg, L.; Colao, M.; Brussee, J.; van der Gen, A. Tetrahedron: Asymmetry 1995, 6, 1123.

12. Amarego, W. Purification of Laboratory Chemicals; Butterworth Heinemann: Burlington, 2003.

13. Klinkmüller, K.-D.; Marschall, H.; Weyerstahl, P. Chem. Ber. 1975, 108, 203.

14. Mandal, A. N.; Raychaudhuri, S. R.; Chatterjee, A. Synthesis 1983, 727.

15. Mitsuhashi, K.; Ito, R.; Arai, T.; Yanagisawa, A. Org. Lett. 2006, 8, 1721.

16. Ecke, G. G.; Napolitano, J. P. J. Am. Chem. Soc. 1955, 77, 6373.

17. Malosh, C. F.; Ready, J. M. J. Am. Chem. Soc. 2004, 126, 10240.

18. Carnduff; J.; Leppard, D. G. J. Chem. Soc., Perkin Trans. 1 1977, 1325. 